\title{
Function, Proteomic and miRomic Study of a Dry Immersion Model of Microgravity implicates the Wnt/Dkk1/AXIN1 axis in Prothrombotic Platelet Phenotype
}

\section{Laura Twomey}

Technological University Dublin

Nastassia Navasiolava

Univ Angers, CHU Angers, CRC, Inserm, CNRS, MITOVASC, SFR ICAT, F-49000 Angers, France

Adrien Robin

Univ Angers, CHU Angers, CRC, Inserm, CNRS, MITOVASC, SFR ICAT, F-49000 Angers, France

Marie-Pierre Bareille

Medes (France)

Guillemette Gauquelin-Koch

Centre National d'Études Spatiales (CNES), Paris, France

Arnaud Beck

Medes (France)

Françoise Larcher

CHU Angers, Laboratoire de Biochimie, Univ Angers, F-49000 Angers, France

Gerardene Meade-Murphy

Department of Pharmacology and Therapeutics, University College Cork, Cork, Ireland

Sinead Sheridan

Department of Sports Science \& Physical Education, The Chinese University of Hong Kong

Patricia Maguire

Conway-SPHERE Research Group, Conway Institute, University College Dublin, Dublin, Ireland

Michael Harrison

Department of Sport and Exercise Science, Waterford Institute of Technology, Cork Road, Waterford, Ireland

\section{Bernard Degryse}

Centre for Preventive Medicine, Dublin City University, Glasnevin, D9, Ireland

Niall Moyna

Centre for Preventive Medicine, Dublin City University, Glasnevin, D9, Ireland

Claude Gharib

Institut NeuroMyoGène, Faculté de Médecine Lyon-Est, Université de Lyon, Lyon, France.

Marc-Antoine Custaud 
Univ Angers, CHU Angers, CRC, Inserm, CNRS, MITOVASC, SFR ICAT, F-49000 Angers, France

Ronan Murphy ( $\square$ ronan.murphy@dcu.ie)

Centre for Preventive Medicine, Dublin City University, Glasnevin, D9, Ireland

\section{Research Article}

Keywords: Platelets, Microgravity, Physical Inactivity, Cardiovascular Disease, Thrombosis, Epigenetics, microRNA, Protein Biomarkers

Posted Date: July 13th, 2021

DOl: https://doi.org/10.21203/rs.3.rs-610421/v1

License: (9) This work is licensed under a Creative Commons Attribution 4.0 International License. Read Full License 


\section{Abstract}

Objective: Ground based research modalities of microgravity have been proposed as innovative methods to investigate the aetiology of chronic age-related conditions such as cardiovascular disease. Dry Immersion (DI), has been effectively used to interrogate the sequelae of physical inactivity (PI) and microgravity on multiple physiological systems. Herein we look at the causa et effectus of 3-day DI on platelet phenotype, and correlate with both miRomic and biomarker expression.

Approach and Results: The miRomic profile of platelets is reflective of phenotype, which itself is sensitive and malleable to the exposome, undergoing responsive transitions in order to fulfil platelets role in thrombosis and haemostasis. Heterogeneous platelet subpopulations circulate at any given time, with varying degrees of sensitivity to activation. Employing a DI model, we investigate the effect of acute PI on platelet function in 12 healthy males. 3-day DI resulted in a significant increase in platelet count, plateletcrit, platelet adhesion, aggregation, and a modest elevation of platelet reactivity index (PRI). We identified 15 protein biomarkers whose expression levels were altered after DI. 22 "DI/PI" related miRNA were identified, of which five have potential targets in the Wnt pathway associated with platelet biogenesis and function. These findings are supported by increased circulating Axin1 and DKK1.

Conclusions: Circulating biomarker and miRomic analysis implicates miRNA regulation of Wnt/Dkk1/AXIN1 axis in DI/PI induced primed platelet phenotype. Taken together, these findings highlight platelets as sensitive adaptive sentinels and functional biomarkers of epigenetic drift within the cardiovascular compartment.

\section{Introduction}

Deciphering the aetiology of chronic diseases presents a continual challenge for basic, translational and clinical research. Pertinent to these endeavours is our understanding of cardiovascular disease (CVD) and associated co-morbidities. Efforts in coping with the health burden of CVD requires in depth knowledge of the causative risk factors in order to develop cost-effective preventive, management and treatment strategies. CVD risk factors can track from childhood into adulthood and are strong predictors of subclinical disease in early life [1]. Up to $80 \%$ of CVD may be prevented if modifiable risk factors, e.g. physical inactivity are evaded [2].

The haemostatic system is a complex ancestral pathway which is physiologically adapted to maintain haemostasis and protect vascular integrity. Risk factors perturb homeostasis, contributing to chronic inflammation and CVD in which platelets play a pivotal role. Moreover, platelets have diverse roles and are involved in many inflammatory conditions. Therefore, depending on the physiological context and the environmental architecture, platelet function may be protective or, conversely, contribute to adverse thrombotic and inflammatory outcomes.

Platelets are small, anucleate cells that travel as resting discoid fragments in the circulation. Their average circulating life span is $8-9$ days and they are derived from haematopoietic stem cells (HSC) 
located in the bone marrow niche. Studies have identified the existence of multiple stem cells types within the various bone cellular niches $[3,4]$. Nevertheless, the dynamics and differentiation steps by which HSCs give rise to the diverse cell types are difficult to characterise due to modelling limitations as well as the complexity of the cellular and molecular processes involved $[5,6]$.

Platelet biogenesis, is an finely orchestrated series of cellular processes known as megakaryocytopoiesis and thrombopoiesis. These distinct, non-mutually exclusive processes are driven by temporal, spatial and stochastic patterns of gene expression, thought to be determined by precise epigenetic mechanisms. Epigenetics involves the intricate interplay between genes and the exposome and is fundament to developmental biology. Several recent studies have demonstrated that epigenetic alterations, mostly encompassing DNA methylation, histone tail modifications, and the biogenesis and effector function of microRNAs and other non-coding RNAs (ncRNAs), play integral role in both primary and secondary haemostasis $[5,7,8]$.

Epigenetic mechanisms display a plasticity in that they are amenable to perturbations throughout our lifespan and drive cell proliferation, differentiation, function and adaptation from conception into adulthood. Environmentally induced epigenetic modifications, such as those elicited by nutrition, stress, pollution, medication and physical inactivity, can influence morbidity and mortality associated with chronic illness and numerous adult onset diseases [9].

Epigenetic control of haemostasis and thrombosis via mechanisms such as DNA methylation and histone modification is a new domain. Limitations in this area of research have hindered progress, as analysis of these processes requires DNA, which is lacking in platelets [6]. New studies have implicated several key molecular pathways involved in platelet aggregation as being regulated by DNA methylation. Examples of such biochemical arbiters of platelet phenotype and function include the platelet receptors platelet-endothelial aggregation receptor 1 (PEAR1) [10], protease-activated receptor 4 (PAR4) [11], glycoprotein VI [12], and P2Y12 [13]. Qualitative and quantitative analysis of discernible platelet phenotypes based on multi 'omics' are being associated with a variety of physiological conditions and sensitivity to anti-thrombotic therapeutics $[8,14,15]$. Various platelet derived parameters known as platelet indices are being employed as a measure of platelet heterogeneity and as surrogate index markers of megakaryopoiesis variation [16]. The molecular basis and significance of platelet phenotypic variation remain vague as they do not lend themselves to lex parsimoniae.

Scientific studies to elucidate the impact of the exposome on platelet biogenesis are emerging as exciting and active fields of research. Our understanding of the adaptive mechanisms underlying the physiological, cellular and molecular responses to physical inactivity, such as altered platelet phenotype is vague. Our knowledge of physical inactivity is somewhat indirect and is mainly based on the positive effects of exercise training on the sedentary population [17]. As a sedentary lifestyle is often associated with obesity and overweight, some mechanisms involved in the pathogenesis of physical inactivity are similar to that of obesity such as insulin resistance, hypertension, and increased inflammation [18]. 
One model being explored to decipher the aetiology of CVD is microgravity $(\mu \mathrm{G})$. Gravity is a continual force impacting biology and physiology, with living organisms adapting to alterations across the gravitational continuum. Variations in gravitational levels are perceived at the molecular and cellular levels, inducing adaptive responses that influence dynamic physiological functions. Fighting gravity requires daily physical exercise, thus exposure to $\mu \mathrm{G}$ is associated with enhanced inactivity [19]. $\mu \mathrm{G}$ provides a unique model to study the effects of global enhanced physical inactivity and deconditioning imposed on healthy subjects [17, 20-27]. Dry immersion reproduces most of the effects of microgravity, inducing rapid and profound deconditioning [20,24], similar to those observed in spaceflight $[26,28]$.

To date there have been no in-depth systematic studies on the effect of DI on platelet biogenesis and concomitant phenotype. Herein, we endeavoured to elucidate the impact of physical inactivity and haemoconcentration induced by 3-Day DI on platelet phenotype as well as the cellular and molecular mechanistic alterations implicated in these adaptations.

\section{Methods}

\section{Subjects}

Twelve healthy non-athletic men aged 26 to 39 years (age $32 \pm 1.4$ year, weight $75 \pm 2 \mathrm{~kg}$, height $178 \pm$ $2 \mathrm{~cm}$, BMI $23.6 \pm 0.4 \mathrm{~kg} / \mathrm{m}^{2}$, maximal oxygen uptake $\mathrm{VO}_{2} \max 39 \pm 1.1 \mathrm{~mL} / \mathrm{min} / \mathrm{kg}$, mean \pm SEM) participated in this study. All participants were non-smokers, had no history of cardiovascular or other chronic diseases, were not taking medication prior to the experiment, and received a comprehensive clinical assessment. All subjects were informed about the experimental procedures and gave their written consent. The experimental protocol conformed to the standards set by the Declaration of Helsinki and was approved by the local Ethic Committee (CPP Sud-Ouest Outre-Mer I, France) and French Health Authorities (n ID RCB: 2014-A 00904 - 43).

\section{General DI protocol}

The study was conducted at the MEDES space clinic, Toulouse, France. General DI protocol is described in detail previously [29]. Briefly, protocol included three days of ambulatory baseline measurement before immersion (B-3, B-2, B-1), three days (72 hours) of dry immersion (DI1, DI2, DI3) and two days of ambulatory recovery $(R 0, R+1)$. The subjects were asked not to exercise during the 8 days of the experiment. Two subjects in two separate baths underwent DI simultaneously. Thermoneutral water temperature $\left(32.5-33.5^{\circ} \mathrm{C}\right)$ was continuously maintained. Light-off period was set at 23:00-07:00. Daily hygiene, weighing and some specific measurements required extraction from the bath. During these short out-of-bath periods, subjects maintained the $-6^{\circ}$ head-down position ("strict" protocol). Total out-of-bath supine time for the $72 \mathrm{~h}$ of immersion was $4.7 \pm 0.16 \mathrm{~h}$. Otherwise, during DI, subjects remained immersed in a supine position for all activities and were continuously observed by video monitoring. Blood pressure (BP), heart rate (HR), and body weight were measured daily at 07:00. Leg echography has been performed daily to ensure the absence of thrombophlebitis. Onset and end of immersion both occurred at 09:00, 
therefore morning measurements and samplings on DI1 were performed before immersion, and on day R0 - still under immersion. Water intake was ad libitum and diet was the same for all participants (standardized to body weight in energy and nutrients). Daily caloric intake was approximately $2820 \mathrm{kcal}$ for baseline and recovery and $2270 \mathrm{kcal}$ for the immersion period. Daily intake for sodium and potassium was approximately 3-4 g. This 3-day DI allowed for several studies on different domains performed by 8 research groups $[25,27,29-33]$.

\section{Blood sampling}

Antecubital venous blood samples (trisodium citrate tube) were collected in the morning before breakfast, before DI (B-3; Pre), under DI on RO (70h of DI; End) and after DI on R + 1 (22h following the accomplishment of 3-day Dl; Recovery). Schematic of the blood sampling protocol is outlined in Table S1 (Supplementary Material).

\section{Blood count and Platelet indices}

Immediately after blood sampling, complete blood count and "platelet count" was performed using the Sysmex XN-3000 ${ }^{\text {TM }}$ Haematology system. Blood count included RBC, WBC, Hb, Hct. Platelet indices included Platelet count (PLT), plateletcrit (PCT), platelet distribution width (PDW), mean platelet volume (MPV), platelet large cell ratio (PLCR). (www.sysmex.co.uk).

\section{Preparation of platelets}

The preparation of Platelet Rich Plasma, Platelet Free Plasma and leukocyte (CD45) depletion of platelets was as previously described [34]. CD45 depletion of platelets for RNA analysis was carried out using EasySep ${ }^{\text {TM }}$ magnetic technology (StemCell). A detailed description with adaptions is outline in Supplementary Material.

\section{Plasma volume estimation}

Percent change in plasma volume on $\mathrm{RO}$ and $\mathrm{R}+1$ vs. $\mathrm{B}-3$ was calculated using $\mathrm{Hb}$ and $\mathrm{Hct}$ count (Dill and Costill formula): DPV $(\%)=100 \times[\mathrm{HbB}(1-0.01 \mathrm{Hcti})] /[\mathrm{Hbi}(1-0.01 \mathrm{HctB})]-100$, where $\mathrm{HbB}$ and HctB are baseline $\mathrm{Hb}$ and $\mathrm{Hct}$ levels, and $\mathrm{Hbi}$ and $\mathrm{Hcti}$ are $\mathrm{Hb}$ and $\mathrm{Hct}$ on days $\mathrm{RO}$ and $\mathrm{R}+1$, respectively.

\section{Platelet-free plasma isolation}

Platelet free plasma (PFP) was isolated for microvesicle work. In order to generate PFP, a double centrifugation method was employed. Blood was drawn using a $21 \mathrm{G}$ needle into a sodium citrate vacutainer $(0.32 \% \mathrm{v} / \mathrm{v}$ final concentration). As standard procedure, the first $3 \mathrm{mls}$ of blood was discarded to avoid contamination from tissue damage due to venepuncture. The blood sample was mixed by gentle inversions to ensure even distribution of the anticoagulant followed by centrifugation at $1550 \mathrm{xg}$ for 20 minutes at room temperature $\left(20-22^{\circ} \mathrm{C}\right)$ to pellet the cells. The supernatant PFP was carefully aspirated leaving a layer of approximately $0.5 \mathrm{~cm}$ undisturbed on top of the cells. The collected PFP was centrifuged again at $13,000 \times$ g for 2 minutes. The PFP was then collected, leaving $20 \%$ of the sample at 
the bottom of the tube. The PFP was separated in $250 \mu$ aliquots and stored at $-80^{\circ} \mathrm{C}$ until further analysis, at which point it was thawed on ice.

\section{Human protein biomarker assay - Proseek multiplex immunoassay}

Proseek biomarker assays were undertaken in collaboration with Olink, Sweden (www.olink.com). Proseek® multiplex CVD II ${ }^{96 \times 96}$ \& Proseek® multiplex inflammation I ${ }^{96 \times 96}$ are high-throughput multiplex immunoassays, each enabling analysis of 92 CVD- or inflammation-related protein biomarkers using $1 \mu \mathrm{L}$ of sample and across samples simultaneously. This high level of multiplexing is achieved by proximity extension assay (PEA) technology. A pair of oligonucleotide-labelled antibodies specific for each biomarker are allowed to pair-wise bind to each target protein in the sample allowing for detection and quantification by real time PCR. Proseek assays were performed by Olink Bioscience (Upsala, Sweden) to evaluate the expression of 152 CVD and inflammatory biomarkers in the PFP samples. Briefly, $1 \mu$ of each sample or negative control was incubated with the conjugated antibodies at $4^{\circ} \mathrm{C}$ overnight. The PEA mixture was added and the products were extended and pre-amplified using PCR (ABI 2720 Thermal cycler, Life Technologies). The detection reagent was added to $2.8 \mu$ of the extended and pre-amplified product, mixed and then loaded into the Fluidigm Gene Expression 96x96 Dynamic arrays (Fluidigm Corporation). The chip was primed using Fluidigm IFC controller HX and loaded into a Fluidigm Biomarker system. Detection and sample analysis was performed using the Fluidigm ${ }^{\circledR}$ BioMark $^{\mathrm{TM}} \mathrm{HD}$ System. This PCR platform enables simultaneous detection of 96 analytes in 96 samples creating 9,216 data points from a single run.

\section{Proseek Multiplex Immunoassay: Data Analysis}

Raw data was analysed using Fluidigm PCR software as per manufacturers guidelines. Briefly, generated Cq values and data was normalized using the extension control and a background value. The data used for statistical analysis was expressed on a log2 scale, where a high value corresponded to a high protein expression. The limit of detection (mean negative control plus 3 x standard deviation) was determined per biomarker per sample. The data was normalised and analysed using GenEx software (MultiD, Gothenburg, Sweden). All statistical analyses (dynamic principal component analysis and one-way ANOVA) were performed on normalized data.

\section{miRNA isolation and amplification}

All RNA procedures were undertaken using the strictest sterile techniques using appropriate RNase free consumables and reagents. The mirVana ${ }^{\circledR}$ RNA extraction kit was used for isolation and purification of total RNA from platelet samples as per manufacturer's instructions. Platelets were prepared as previously outlined and pelleted by centrifugation at 2000xg for 12 minutes at RT. 400 $\mu$ l of total lysis binding solution was added to the cells at the commencement of the protocol. The total RNA was then eluted into a fresh collection tube by centrifugation at 10,000xg for 30 seconds using $100 \mu$ of elution buffer, which was pre-heated to $95^{\circ} \mathrm{C}$. The RNA was analysed on a NanoDrop ${ }^{\circledR}$ Spectrophotometer and samples stored at $-80^{\circ} \mathrm{C}$. 


\section{Platelet microRNA and profiling}

Platelet miRNA profiles from 8 out of the 12 subjects (due to sample limitations) were assessed at the Pre-DI and End-DI time points (B-3 and R0). Total (non-normalized) leukocyte-depleted platelet RNA (ranging from 3.5 to $7.0 \mathrm{ng}$ ) was extracted from equal volumes of platelet rich plasma using the miRVANA RNA extraction kit. The miRNA profile was determined by RT-qPCR using Applied Biosystems OpenArray ${ }^{\circledR}$ plate technology on the QuantStudio ${ }^{\text {TM }} 12 \mathrm{~K}$ Flex Real-Time System. For a complete miRNA profile, 754 human miRNAs were quantified.

miRNA profiling was carried out using the Applied Biosystems ${ }^{\circledR}$ TaqMan® Low Density Array (TLDA) Human miRNA A (v2.0) and B (v3.0) cards set. For analysis on the TLDA cards, total RNA was firstly extracted from platelets using the Ambion $^{\mathrm{Tm}}$ mirVana ${ }^{\circledR}$ miRNA isolation kit as per manufacturer's instructions. Single stranded cDNA was synthesised from total platelet RNA using the Applied Biosystems TaqMan ${ }^{\circledR}$ miRNA Reverse Transcription (RT) Kit. For a full miRNA profile two RT reactions were needed incorporating primers for both pool A and B miRNA panels. The RT reaction had a final volume of $7.5 \mu \mathrm{l}$ and contained: $3 \mu \mathrm{l}(1-350 \mathrm{ng})$ total RNA and $4.5 \mu \mathrm{l}$ of RT master mix. As the total RNA yield from platelets is generally lower than nucleated cells (less than $350 \mathrm{ng}$ ), a preamplification step was carried out prior to committing the cDNA to the TaqMan miRNA arrays to uniformly pre-amplify desired cDNA prior to quantification with the TLDA cards. The sample was diluted with $75 \mu \mathrm{l}$ of $0.1 \mathrm{x}$ TE buffer

$(\mathrm{pH} 8)$ and used immediately for array analysis or stored for up at $-80^{\circ} \mathrm{C}$ for future use.

A 7900HT PCR system was used for initial miRNA profiling and a QuantStudio ${ }^{\text {TM }}$ 12K Flex Real-Time PCR system was used for large scale profiling. DNA polymerase from the TaqMan ${ }^{\circledR}$ Universal PCR Master Mix amplifies the target cDNA using sequence-specific primers and a probe on the TaqMan microRNA array. $100 \mu \mathrm{l}$ of the master mix was dispensed into each chamber of the array. The card was centrifuged twice for 1 minute at $1000 \times \mathrm{g}$ to fill each of the 384 wells of the card in an Eppendorf 5810R centrifuge. OpenArray ${ }^{\circledR}$ plate technology on the QuantStudio ${ }^{\text {TM }} 12 \mathrm{~K}$ Flex Real-Time System (Paris, France) was used for large scale miRNA profiling.

\section{Bioinformatics analysis of platelet microRNA data}

Bioinformatics methods were used to extrapolate biological meaning from miRNA that were significantly up or downregulated after the DI. First, microRNA target prediction was performed using online software tools Targetscan (http://www.targetscan.org/vert_71/) and microRNA.org. Following this, involvement of these targets in pathways of interest from the Kyoto Encyclopaedia of Genes and Genomes (KEGG) was analysed using the Database for Annotation, Visualization and Integrated Discovery (DAVID) (http://david.niaid.nih.gov).

\section{Platelet function assay with Impact-R cone and plate analyser}


The Impact-R device tests platelet function in anticoagulated whole blood under near physiological conditions. An image analyser measures the adhered platelets and results are expressed as a percentage of the well surface covered (SC\%) as an index of platelet adhesion, and average size of the aggregates $\left(\mathrm{AS} \mu \mathrm{m}^{2}\right)$ as an index of aggregation. Blood samples drawn into sodium citrate vacutainers were analysed 1 hour post-draw as per manufacturers recommendations. Blood samples were mixed for 1 minute at $10 \mathrm{rpm}$ prior to loading on the apparatus. A $130 \mu \mathrm{l}$ aliquot of the blood sample was applied to the centre of the well followed immediately by the bell housing and cone on top. The selected programme was started (arterial shear rate of 1800 per second for 2 minutes). Platelet were stained with $500 \mu \mathrm{l}$ of May Grunwald stain solution for 1 minute. Images were then captured at random. Seven images were captured in total. The software analysed the captured images by eliminating the four least readable images and calculating the average of the remaining three. Results were expressed as SC and AS with a visual and graphical result provided for each test.

\section{VASP/P2Y 12 phosphorylation assay}

Flow cytometry experiments in this thesis were performed on a Accuri C6 (BD Biosciences). To ensure day-to-day sample reproducibility, all cytometers were calibrated daily. To determine the VASP phosphorylation state of whole blood, an adapted standardized flow cytometric assay (BioCytex, France) was employed [35]. The VASP-P analysis was performed within $4 \mathrm{~h}$ after blood collection as per manufacturer's instructions.

\section{Microvesicle quantification and analysis}

The NanoSight NS300 and Syringe Pump were used to quantify microvesicles (exosomes and microparticles) in PFP samples. Nanoparticle tracking analysis technology (NTA) used in this device combines the properties of light scattering and Brownian motion to attain measurements including concentration and size distribution of particles in a liquid suspension (Methods; Supplementary Material).

\section{Statistical analysis}

Results are expressed as mean \pm SEM. Statistical comparisons were performed using a variety of tests, depending on the experimental procedure. Main tests included independent t-tests, paired samples t-tests, repeated measures ANOVA and one-way ANOVA/one-way ANCOVA. Pearson product coefficient was used to examine relationships between variables. Statistical significance was set at a level of 0.05 . SPSS v19 statistical package was used to analyse results.

\section{Results}

\section{Effect of dry immersion on physiological and haematological characteristics}


The overview of physiological responses to this 3-day dry immersion protocol has previously been reported [29]. Overall, DI was well tolerated, with no dropouts. Moderate back pain was reported at the beginning, as it is usually seen in dry immersion protocols. HR and BP remained within normal limits throughout the protocol. HR rose slightly at END-DI ( $57 \pm 3 \mathrm{bpm})$ and RECOVERY-DI ( $57 \pm 2 \mathrm{bpm})$ vs. PREDI (53 \pm 2 bpm). SBP and DBP were not significantly modified (Figure S1; supplementary material). Body weight during immersion decreased by approximately 1-2kg (Figure S1). Daily echo-doppler of the lower limbs did not reveal venous problems/thrombosis. Estimated plasma volume showed a $14 \pm 2 \%$ decrease at END-DI vs. PRE-DI $(p<0.0001)$. At RECOVERY-DI plasma volume did not significantly differ from PRE$\mathrm{DI}(+4 \pm 2 \%, \mathrm{p}=0.103)$. Changes in haematological parameters in response to $\mathrm{DI}$ are shown in Fig. 1 . There was a significant increase in WBC, RBC concentration, HGB and HCT at the end time point. There was a significant decrease in WBC, RBC concentration and HCT between post and recovery, almost to basal levels.

\section{Effect of dry immersion on platelet indices}

There were no changes in platelet large cell ratio, mean platelet volume and platelet distribution width between any time points. There was a significant increase in platelet count and plateletcrit from PRE-DI to END-DI and subsequently, a significant decrease in these parameters from END-DI time point to RECOVERY-DI (Fig. 2).

\section{Effect of dry immersion on platelet function}

Figure 3A and 3B (representative images from Impact-R (i) PRE-DI, (ii) END-DI and (iii) RECOVERY-DI show the effect of physical inactivity on platelet function. There was a significant increase in platelet adhesion post-immersion and a subsequent decrease between the post and recovery time points. There was also a significant increase in platelet aggregation from PRE-DI to END-DI.

\section{Effect of dry immersion on platelet VASP phosphorylation}

To assess if basal platelet VASP phosphorylation was affected by physical inactivity, the standardised $\mathrm{P}_{2} \mathrm{Y}_{12}$ /VASP kit was used in a flow cytometry assay. The platelet reactivity index (PRI) represents changes in VASP phosphorylation. There was a small insignificant increase in PRI post DI (Figure S2). There were individual fluctuations in VASP phosphorylation during the DI, possibly reflective of interindividual variability due to genetic architecture.

\section{Effect of dry immersion on protein biomarker expression}

Protein biomarker expression of platelet poor plasma (PPP) was assessed using the Cardiovascular and Inflammatory protein biomarker panels (Olink Bioscience). The levels of 15 proteins differed significantly between time points (Table 1). Results are expressed as normalised protein expression (NPX) on a log2 scale. Therefore, a normalised increase of 1 is equal to a two-fold increase in protein amount. For 15 proteins out of 131 detected, expression levels differed significantly between different stages of the DI 
protocol. Seven of these proteins affected by DI are related to platelet function, namely Heat shock protein 27 (HSP27), Lectin-like oxidised LDL receptor (LOX-1), NF-Kappa-B essential modulator (NEMO), Proto-oncogene tyrosine protein kinase (SRC), Dickkopf-related protein (DKK1), Axin1, SIRT2, and IL-6. The changes in biomarker expression levels are displayed in Figs. 4 and 5.

Table 1

Protein biomarkers which were differentially expressed after the DI. The column on the left displays differentially expressed proteins from the CVD panel and the column on the right displays differentially expressed proteins from the inflammation panel.

\begin{tabular}{|c|c|}
\hline $\begin{array}{l}\text { Cardiovascular Biomarker Panel } \\
\text { (Protein biomarker \& Main function) }\end{array}$ & $\begin{array}{l}\text { Inflammatory Biomarker Panel } \\
\text { (Protein biomarker \& Main function) }\end{array}$ \\
\hline Adrenomedullin (ADM) & Axin-1 (AXIN1) \\
\hline $\begin{array}{l}\text { Vasodilation and regulation of hormone } \\
\text { secretion }\end{array}$ & Negative regulator of the WNT signalling pathway \\
\hline Dickkopf related protein-1 (DKK1) & Interleukin-6 (IL6) \\
\hline WNT signalling pathway inhibitor & Pro-inflammatory cytokine \\
\hline Heat shock protein-27 (HSPB1) & STAM-binding protein (STAMBP) \\
\hline Stress resistance, actin organization & Cytokine-mediated signalling \\
\hline Lectin like oxidised LDL receptor-1 (OLR1) & Sulfotransferase 1A1 (SULTA1) \\
\hline Binds, internalises, degrades oxidized $L D L$ & $\begin{array}{l}\text { Catalyse the sulphate conjugation of hormones, } \\
\text { neurotransmitters }\end{array}$ \\
\hline NF-Kappa B essential modulator (IKBKG) & SIRT2 \\
\hline Inflammation, immune genes & NB: Possible role in epigenetic gene silencing \\
\hline Renin (REN) & Matrix metalloproteinase-10 (MMP10) \\
\hline Activation of angiotensinogen pathway & Degradation of extracellular matrix \\
\hline \multirow{2}{*}{$\begin{array}{l}\text { Proto-oncogene non receptor tyrosine } \\
\text { kinase (SRC) }\end{array}$} & Matrix metalloproteinase - 3 (MMP3) \\
\hline & Degradation of extracellular matrix \\
\hline \multicolumn{2}{|l|}{ Tissue plasminogen activator (PLAT) } \\
\hline Disintegration of blood clots & \\
\hline
\end{tabular}

\section{Effect of dry immersion on platelet poor plasma microvesicles}

Nanosight technology was employed to qualitatively and quantitatively interrogate platelet poor plasma (PPP) for MV size and concentration at each time point. A slight but non-significant decrease in the 
average MV size and a modest increase in average MV concentration was observed at END-DI (post-DI time point). For further analysis, MVs were divided into three categories: exosomes, microparticles and large microparticles with modest increases in each category END-DI, again however the changes were not statistically different (Figure S3).

\section{Effect of dry immersion on platelet microRNA expression}

miRNA expression profiles were analysed from non-normalized leukocyte-depleted platelet RNA (ranging from 3.5 to $7.0 \mathrm{ng}$ ) extracted from equal volumes of platelet rich plasma from 8 of the 12 subjects at the time points PRE-DI and END-DI. Western blots of the miRNA regulatory proteins Dicer, Argonaute 2 and Drosha demonstrated the presence of both Dicer and Ago2 with the absence of Drosha (data not shown), in line with previous published studies. The number of miRNA either up or downregulated END-DI on the A and $B$ array cards are shown in Figure S4. Heat maps comprised of the most highly expressed miRNA were then constructed for both the A and B panel of miRNA. This allowed visualisation of miRNA expression profiles between subjects, before the DI had been implemented. This is shown in Figures $S 5(A) \& S 5(B)$. A shift in colour from red to blue indicates decreasing expression of that miRNA.

\section{Identification of miRNA affected by physical inactivity}

By comparing the miRNA expression profiles of the subjects between PRE-DI and END-DI, we identified 22 significantly differentially expressed miRNA with a fold change of more than 1.2 (12 upregulated and 10 downregulated). Most reported miRNA fold changes are small ( 1.5 fold). The miRNAs that were differentially expressed on the A card are shown in Fig. 7A \& 7B, while the miRNA differentially expressed on the B card are shown in Fig. 7C \& 7D.

\section{Bioinformatic analysis of differentially expressed miRNA}

Bioinformatic interrogation was employed to ascribe biological relevance of the significantly differentially expressed miRNA END-DI. In silico analysis was used to determine putative targets of these identified miRNA of interest. A comprehensive approach was employed using complementary online software tools including Targetscan and microRNA.org. Subsequent pathway analysis was undertaken based on the Kyoto Encyclopaedia of Genes and Genomes (KEGG) and accessed using the DAVID bioinformatics database. A table was constructed to illustrate the number of potential genes the miRNA could target and the corresponding KEGG pathways common to a number of differentially expressed miRNA which are involved in platelet function (Table S2 - Wnt signalling, cytoskeletal dynamics, ECM-Receptor interactions and Toll like receptor signalling).

\section{KEGG pathway analysis}

Maps of key pathways involved in platelet function and activation were downloaded from the KEGG database using DAVID. Pathways were chosen based on their involvement in platelet function and signalling, specifically in adhesion and aggregation, but also their inflammatory potential. Included in these were the Wnt signalling pathway, regulation of actin cytoskeleton, ECM interaction and Toll-like 
receptor pathway, of which the Wnt signalling pathway appeared repeatedly in both the up and downregulated miRNA targets. Genes that were potential targets for multiple miRNAs were circled red, genes that were targets for a single miRNA were circled yellow. Potential miRNA targets of genes involved in the Wnt signalling pathway are shown in Figure S6, potential miRNA targets of genes involved in the reorganisation of the actin cytoskeleton pathway are shown in Figure S7 and potential miRNA targets of genes involved in the ECM receptor-interaction pathway are shown in Figure S8.

\section{miRNA targets of the Wnt signalling pathway}

The Wnt signalling pathway involved the largest number of differentially regulated miRNA post $\mathrm{DI}$, as shown previously in Table 2. The protein biomarkers Axin 1 and DKK1 were also differentially expressed END-DI. We used online databases to determine additional miRNA which could target Axin1 and DKK1 (Fig. 7A \& 7B). The combined action of multiple down or upregulated miRNA could have affected the gene and subsequent protein expression of Axin1 and DKK1.

Table 2

Involvement of differentially regulated miRNA in KEGG cell pathways. Bioinformatics of differentially regulated miRNA. Table shows KEGG pathways common to a number of differentially expressed miRNA and which are involved in platelet function.

\begin{tabular}{|c|c|c|c|c|c|c|}
\hline \multicolumn{7}{|c|}{ KEGG pathway } \\
\hline \multicolumn{3}{|c|}{ Wnt signalling } & \multicolumn{2}{|c|}{$\begin{array}{l}\text { Regulation of Actin } \\
\text { cytoskeleton }\end{array}$} & \multirow{2}{*}{$\begin{array}{l}\text { ECM receptor } \\
\text { interaction } \\
\text { miR-143 }\end{array}$} & \multirow{2}{*}{$\begin{array}{l}\text { Toll like } \\
\text { receptor } \\
\text { miR-143 }\end{array}$} \\
\hline miR-888 & $\begin{array}{l}\text { miR- } \\
500\end{array}$ & $\begin{array}{l}\text { miR- } \\
145\end{array}$ & miR-143 & miR-34a* & & \\
\hline $\begin{array}{l}\text { miR- } \\
299 a-5 p\end{array}$ & $\begin{array}{l}\mathrm{miR}- \\
203\end{array}$ & $\begin{array}{l}\operatorname{miR}- \\
106 b^{*}\end{array}$ & miR-199a & miR-744 & miR-302a & miR-15a \\
\hline miR-484 & $\begin{array}{l}\mathrm{miR}- \\
302 \mathrm{a}\end{array}$ & $\begin{array}{l}\operatorname{miR}- \\
34 a^{*}\end{array}$ & miR-500 & miR-494 & miR-199a & miR-34a* \\
\hline miR-199a & $\begin{array}{l}\text { miR- } \\
190 b\end{array}$ & $\begin{array}{l}\text { miR-24- } \\
2\end{array}$ & miR-302a & miR-15a* & miR-484 & \\
\hline miR-200a & $\begin{array}{l}\operatorname{miR}- \\
15 a^{*}\end{array}$ & & miR-374a & miR-106b* & & \\
\hline
\end{tabular}

\section{Discussion}

Despite the strong links between physical inactivity (PI) and CVD risk [1], there remains a knowledge gap into the effects of PI in healthy subjects. This study allowed a unique opportunity to investigate the effects of acute PI on platelet phenotype. Exposure to $\mu \mathrm{G}$ induces a constellation of adaptive physiological modifications, and in particular the cardiovascular system. DI is characterised by enforced physical inactivity [36]. During DI, the signs of muscle disuse, bone remodelling and spine changes, extensive cardiovascular deconditioning and other alterations mimic the adaptation observed in astronauts. CV deconditioning is a state whereby the CV system does not react efficiently to challenge, 
distinguished by a reduced capability for exercise, orthostatic intolerance and tachycardia [24]. Initial reactions to DI occur in the first 12 hours and are caused by immediate modifications in body fluid distribution and a removal of support structure [20-23].

In the 1980's, Kirichenko et al. demonstrated that 7 days of DI induced a significant increase in platelet count and hyper-coagulopathic changes to platelet hemostasis [37, 38]. A 2011 study examined the effects of simulated microgravity on the miRNA profile of human lymphoblastic cells using a high aspect ratio vessel to model microgravity in space [39]. More recently, 42 miRNAs from cultured human blood lymphocytes from 12 healthy subjects were differentially expressed in microgravity stimulated cells compared to static cells, with resultant mRNA gene targets involved in inflammatory and apoptotic responses [40]. Malkani et al. elucidated the role of circulating microRNAs as both a potential biomarker for health risks associated with spaceflight and a countermeasure to mitigate the damage caused to the body by the space environment [41].

\section{Haematological parameters}

Both spaceflight and its proxies result in an initial plasma volume decrease (approximately $10-15 \%$ ) after which it remains stable $[22,42]$. We noted a $14 \%$ decrease in plasma volume post DI, with return to baseline at the recovery time point. Blood viscosity usually increases due to the decrease in plasma volume, with a corresponding decrease in RBC is required to maintain blood viscosity [43]. However, decreases in RBC due to altered erythropoiesis takes time, so haemoconcentration was still present at the end of immersion (END-DI), reflected by an increase in RBC. There were also significant increases in $\mathrm{Hb}$ and HCT during the immersion, although these values remained within the normal limits for healthy individuals. These findings were as expected, as lower plasma volume would result in increased concentrations of RBCs. In general, real and simulated microgravity results in changes to physical properties of RBCs [20,44]. Both a 7-day DI [45] and 5-day DI [46] resulted in an increase in RBCs and altered morphological composition of red blood in healthy males. Navasiolava et al. reported a significant increase in RBC, $\mathrm{Hb}$ and HCT after seven days of DI, which returned to normal after recovery [23]. Unlike our study, they did not observe a significant difference in WBC. However, Bedendeeva et al. noted a $40 \%$ increase in leukocytes after DI [47].

\section{Platelet indices}

The effects of DI on platelet indices were investigated as primary indicators of platelet phenotype, and also as surrogate markers of altered megakaryocytopoiesis (Fig. 2). There was an increase in platelet count (PLT) between PRE-DI to END-DI time points, probably reflecting the loss of plasma volume during the DI. There was a significant decrease in PLT between END-DI and RECOVERY-DI suggesting that platelet count returned to pre-immersion levels once subjects began to resume ambulatory activity. Navasiolava et al. found no significant difference in PLT after 7-days DI [22]. Other studies have indicated a significant increase in PLT after 7-day DI [37,38]. There were no changes in MPV, PDW or PLCR after three days of DI. There was a significant increase in plateletcrit from PRE-DI to END-DI and a significant decrease in plateletcrit from END-DI to RECOVERY-DI. Plateletcrit appears to be one of the more sensitive 
platelet indices markers. Overall, the combined increases in PLT and PCT observed at the time-point ENDDI may indicate altered megakaryocytopoiesis.

\section{Platelet function}

The effect of PI on platelet function was quantified using the Impact R cone and plate analyser. We observed a significant increase in platelet adhesion from PRE-DI to END-DI suggesting stronger plateletsurface interactions in response to physical inactivity and DI. There was a significant decrease in SC\% from END-DI to RECOVERY-DI indicating platelet adhesion levels had returned to their basal state. There was a significant increase in AS from PRE-DI to END-DI suggesting that PI/DI results in elevated platelet aggregation with increased activation of GPallb $\beta 3$ and increased affinity for fibrinogen binding. Platelet aggregation decreased slightly from END-DI to RECOVERY-DI. The reduction in blood volume as a consequence of reduced total body water in the body during microgravity has been suggested as a factor for thrombotic tendencies, which could have been a determinant of increased platelet activation in this study [48].

PI causes endothelial dysfunction as well as increasing soluble von Willebrand factor (vWF), tissue factor (TF) expression and endothelial microparticle production, facilitating platelet activation under disturbed flow, inferring that altered shear stress may not activate platelets directly [49]. Haemoconcentration, body fluid redistribution, hypodynamia experienced during DI may increase the risk of thrombosis and could also have contributed to enhanced platelet adhesion and aggregation observed after the DI [20, 23]. The increase in blood viscosity after 24 hours of DI may have been a contributory factor to the platelet hyperaggregability [50]. The use of a viscometer and in parallel with platelet function tests could provide informative data [51]. Interestingly, Kuzichkin et al. observed an increase in plasma fibrinogen concentration after short-term space flights and 7-day DI [52]. Assessment of fibrinogen levels and allb $\beta 3$ activation levels would provide an insight into the increased platelet aggregation END-DI [53].

\section{Platelet VASP phosphorylation}

VASP is an intracellular regulator of actin dynamics in platelets and plays a key role in regulating platelet adhesion and aggregation [54]. VASP is phosphorylated by CAMP- and cGMP-regulated protein kinases and reflects with inhibition of platelet activation, inhibition of allb $\beta 3$ and a restriction of VASP to bind to F-actin [55]. Consequently, decreased VASP phosphorylation can result in platelet hyperreactivity. We observed a minor non-significant increase in the average PRI (5\%) at END-DI suggesting a reduction in VASP phosphorylation and increase in platelet activation (Figure S2). Assinger et al. showed that VASP phosphorylation at basal levels on the Ser239 residue was significantly reduced in smokers [56]. Employing both picomolar and nanomolar concentrations of $\mathrm{PGE}_{1}$, smokers demonstrated a reduced VASP phosphorylation correlating with increased P-selectin expression. However, at maximal PGE $_{1}$ concentrations traditionally used for in vitro assays, no difference between smokers and non-smokers was noted. As the VASP/P2Y 12 kit uses a maximal effective doses of $\mathrm{PGE}_{1}$, the PRI was similar (i.e. no statistical difference) between the smokers and the non-smokers. This experimental observation highlights the importance of employing a range of $\mathrm{PGE}_{1}$ concentrations during assay development and 
emphasises the assessment of VASP phosphorylation in the presence of submaximal quantities of PGE could be more physiologically informative and relevant. Physical inactivity could produce similar responses and require the same level of investigation.

\section{Protein biomarker expression}

We also examined the effect of PI on platelet poor plasma (PPP) protein biomarkers. The expression profiles of these proteins were analysed at PRE-DI, END-DI and RECOVERY-DI. 131 out of 157 of the proteins were detected in all samples, with 15 proteins significantly $\triangle D E$ between different stages of the DI (Table 1). Key proteins affected by physical inactivity included Heat shock protein 27 (HSP27), Lectinlike oxidised LDL receptor (LOX-1), NF-Kappa-B essential modulator (NEMO), Proto-oncogene tyrosine protein kinase (SRC) and Dickkopf-related protein (DKK1) (Fig. 4).

Numerous environmental and physiological stressors mediate the expression of heat shock proteins (HSPs). HSPs have been identified in atherosclerosis [57] and post exercise [58]. HSP-27 was significantly elevated END-DI suggesting a stress response to the effects of acute physical inactivity. HSP27 has been proposed as a key player in actin polymerisation during platelet shape change, and phosphorylated HSP27 is released from human platelets upon collagen activation, and is associated with the acceleration of platelet aggregation [59]. Moreover, ADP induces phosphorylation of HSP27 with resultant platelet activation markers PDGF and SCD40L release [60]. Elevated HSP-27 END-DI indicates that it was in response to the physiological stress of physical inactivity.

Lectin-like oxidised low-density lipoprotein receptor-1 (LOX-1) is a scavenger receptor and is expressed on numerous cells, including platelets, in an activation dependent manner [61]. LOX-1 was significantly elevated END-DI. As LOX-1 recognises and binds to activated platelets, exposure of LOX-1 on the surface of activated platelets might encourage thrombus formation. Furthermore, inhibition of LOX-1 in platelets was shown to prevent platelet aggregation [62]. LOX-1 is associated with obesity and physical inactivity and could represent a marker of platelet activation in response to sedentary behaviour [63].

DKK1 was significantly upregulated post DI. Platelets represent a major source of circulating DKK1, which is an antagonist of the Wnt signalling pathway and is released from platelet a-granules upon activation [64]. Plasma DKK1 levels are significantly higher in disease states including T2DM and atherosclerosis [64]. DKK1 can also influence platelet-mediated endothelial cell activation involving the $\mathrm{Wnt} / \beta$-cat signalling pathway and NF-KB pathways.

A number of inflammatory proteins were also differentially regulated END-DI (Fig. 5). Axin-1 is a key member of the Wnt signalling pathway, acting as a scaffold protein and a negative regulator of the Wnt signalling pathway [65]. As Wnt signalling negatively regulates platelet function and modulates the major platelet receptor GPallb $\beta 3$, an increase in Axin-1 levels could also have contributed to platelet adhesion and aggregation levels in this study [66-68].

IL-6, a pleiotropic inflammatory cytokine, was significantly increased END-DI. IL-6 has been adversely linked with sedentary time in a large study of $>500$ participants aged $\sim 63$ years and at high risk for 
T2DM [69] and is elevated after physical inactivity in our study. While platelets do not express IL-6, it can affect platelet activation [70]. IL-6 is also a potent thrombopoietic factor promoting maturation of human megakaryocytes in vitro [71]. A number of inflammatory and CVD proteins were $\triangle \mathrm{DE}$ after our DI, suggesting that extensive physical inactivity affects both immune and platelet function. Future proteomic studies would prove insightful.

\section{Platelet poor plasma microvesicles}

We investigated the effect of physical inactivity on the number and size of MVs in PPP. Figure S3 summarises the changes in circulating MVs in response to DI. There was no change in average MV size END-DI. We found non-significant increases in overall MV concentration. For separate analysis of MV subpopulations, MVs were divided into three distinct categories; Exosomes (30-100nm), microparticles $(100-255 \mathrm{~nm})$, and large microparticles $(>255 \mathrm{~nm})$. There were non-significant increases in exosome and MP concentrations END-DI. Interestingly, there was a non-significant increase in larger MP concentration END-DI, which could suggest the generation of larger and potentially more procoagulant MPs. Studies have shown that endothelial MP levels increased on the third day of a seven-day DI experiment [22]. As DI results in reduced stroke volume and cardiac output, it was suggested that an endothelial dysfunction to NO and deterioration in hemodynamic conditions could contribute to increases in EMPs. The changes in endothelial vasodilatory capacity could also have resulted in platelet hyperreactivity and increased MV levels in our study. Platelet-derived MVs represent the most abundant MV source (70-90\%) released into blood circulation, and we would expect to see a larger increase in PMPs produced END-DI. Future studies of MVs are warranted.

\section{Platelet microRNA (miRNA)}

To further understand the significance of PI on the molecular mechanisms which underpin platelet phenotype, we investigated miRNA profiles PRE-DI and END-DI, to determine if the miRNome for platelets was altered by physical inactivity. In brief, miRNA are short (18-24) nucleotide long non-coding RNA molecules. They regulate gene expression by hybridising to the 3' UTR of mRNA. The existence and functionality miRNA in the anucleate human platelets has been described and constitute $80 \%$ of all small RNAs in platelets [72], expressing relatively high quantities of miRNA compared to their nucleated counterparts [73]. Platelet miRNA levels have been demonstrated to be associated with phenotype [74].

Platelets expressed a total average of 436 miRNA PRE-DI and 438 END-DI, in line to previous similar published studies [72, 73]. We identified 22 miRNA which were significantly up or downregulated at ENDDI (Fig. 6). 10 miRNA were significantly downregulated and 12 miRNA which were significantly upregulated at END-DI. Interestingly, miR-374a family has been demonstrated to regulate Wnt/beta-catenin signalling [75].

As platelets do not express the miRNA nuclear machinery Drosha and DGCR8, the de novo synthesis of new miRNA in platelets is negligible. Upregulation of miRNA in response to physical inactivity could be derived from the processing of pre-miRNA to mature miRNA or as a reflection of increased levels miRNA in their megakaryocyte precursor. As platelets can release MV-containing miRNA upon activation, 
downregulated miRNA in this study may reflect this process. These findings suggest that platelet miRNAs correlate platelet activation in vitro and may have great potential as biomarkers of $\mathrm{PI}$.

\section{Bioinformatics of differentially expressed DI regulated miRNA}

The fold change, number of conserved targets, and number of pathways the predicted genes are linked to are included in Table 22 for each $\triangle D E$ miRNA. Table 2 shows the miRNA potentially involved in the pathways of interest and whether these specific miRNAs were up or downregulated with DI. Pathways were chosen based on their involvement in platelet function and signalling.

Of major interest from the findings of this study was the predicted involvement of $\triangle D E$ miRNA on the Wnt signalling pathway, as both DKK1 and Axin-1 proteins were differentially expressed END-DI, and are involved in the Wnt pathway. The literature has described roles for Wnt- $\beta$-catenin $[67,68]$, and noncanonical Wnt signalling pathways in platelet function [76]. Recombinant Wnt3a ligand was shown to inhibit platelet adhesion, shape change, dense granule secretion and inhibiting activation of allb $\beta 3$ resulting in decreased platelet adhesion to fibrinogen and subsequently reduced aggregation [67].

DKK1 was one of the significantly upregulated proteins identified from the biomarker panel END-DI and we therefore sought to identify $\triangle \mathrm{DE}$ miRNA that could target DKK1 (Fig. 7A). A number of these miRNA were downregulated in our study, suggesting that a simultaneous downregulation of multiple miRNA targeting DKK1 may act together increasing DKK1 expression. miR-302a, the most down regulated miRNA, targets DKK1. The increase in DKK1 could negatively regulate the Wnt signalling pathway and ultimately, elevated platelet adhesion levels.

Axin-1, a key mediator of the Wnt/ $\beta$-cat pathway, was identified as one of the proteins that was upregulated after the DI [77]. Interestingly, two downregulated miRNAs, miR-203 and miR-200a were identified as potential regulators of Axin-1. Additionally, a number of other miRNA predicted to target Axin1 were also downregulated as shown in Fig. 7B. The simultaneous downregulation of multiple miRNA targeting Axin-1 may explain the observed increase in Axin-1 and result in reduced Wnt signalling in platelets with elevated platelet adhesion and aggregation.

Evidence of physical activity-specific microRNA signatures have seeded the notion that there must also be physical inactivity-specific miRNA profiles [78]. Epigenetic variation could be a potential mechanism allowing for independent or perhaps synergistic effects of physical inactivity on platelet function. Hibler et al. recently described indications for epigenetic variation and miRNA expression as a link between physical activity and sedentary lifestyle [79]. We hypothesised that an epigenetic adaptation to physical inactivity is also present inducing epigenetic changes in megakaryocytopoiesis and altered platelet phenotypes.

\section{Conclusion}


3-day DI induced rapidly reversible shift to primed platelet phenotype in healthy men, reflected by increased adhesion and aggregation. We have identified $15 \triangle D E$ protein biomarkers associated with $\mu \mathrm{G} / \mathrm{PI}$. Their expression trends could be of importance for developing 'biosignatures' of physical inactivity. We also identified $22 \triangle \mathrm{DE}$ platelet miRNA END-DI. These PI related miRNA have potential targets involved in pathways associated with platelet function. It is evident from this study that the identification of unique signatures of several miRNA, rather than a single miRNA in isolation may enhance diagnostic accuracy [80]. The canonical Wnt signalling pathway may signify a novel endogenous mechanism for regulating platelet activity in response to physical inactivity. Collectively, our results provide evidence for the early and robust deleterious impact of reduced daily activity on platelet function and phenotype, highlighting the vulnerability of the vasculature to a sedentary lifestyle. It also highlights the importance of physical activity and exercise medicine.

\section{Abbreviations}

ADP - Adenosine Diphosphate; AS $\mu m^{2}$ - Aggregate Size in $\mu \mathrm{m}^{2}$ units; BMI - Body Mass Index; CAMP Adenosine 3',5'-cyclic monophosphate; CFU-Mk - Colony-Forming Unit (CFU) assays of Megakaryocyte Progenitors; cGMP - Guanosine 3,5-cyclic monophosphate; CVD - Cardiovascular Disease; DBP - Diastolic

Blood Pressure; DI - Dry Immersion; DKK1 - Dickkopf-related protein 1; ECM - Extracellular Matrix; EMP Endothelial derived Microparticles; $\mathrm{Hb}$

Haemoglobin; HCT-Haematocrit; HR - Heart Rate; HSC - Haematopoietic Stem Cells; HSP27 - Heat shock protein 27; IL6 - Interleukin-6; KEGG - Kyoto Encyclopaedia of Genes and Genomes; LOX-1 - Lectin-like oxidised low-density lipoprotein receptor-1; MFI - Mean Fluorescent Intensity; miRNA - microRNA; MP Microparticles; MPV - Mean Platelet Volume; MV-Microvesicles; ncRNA - noncoding RNA; NEMO NFKappa-B Essential Modulator; NPX - Normalised Protein Expression; NTA - Nanoparticle Tracking Analysis; PAR4-Protease Activated Receptor 4; PCT-Plateletcrit; PDW - Platelet Distribution Width; PEA Proximity Extension Assay; PEAR1 - Platelet-Endothelial Aggregation Receptor 1; PFP - Platelet Free Plasma; PGE $_{1}$ - Prostaglandin $E_{1}$; PI - Physical Inactivity; PLCR - Platelet Large Cell Ratio; PLT - Platelet count; PPP - Platelet Poor Plasma; pre-miRNA - Preliminary miRNA; pri-miRNA - Primary miRNA; PRI Platelet Reactivity Index; PRKAR2B - Protein Kinase CAMP-Dependent Type II Regulatory Subunit Beta; PRP - Platelet Rich Plasma; RBC - Red blood cell count; SBP - Systolic Blood Pressure; SC\% - Surface Covered; SIRT2 - Sirtuin 2; SRC - Proto-oncogene tyrosine protein kinase; T2DM - Type 2 Diabetes Mellitus; TAC - Tetrameric Antibody Complex; TF - Tissue Factor; VASP - Vasodilator-Stimulated Phosphoprotein; $\mathrm{VO}_{2}$ max - Maximal Oxygen Uptake; WBC - White blood cell count; $\triangle \mathrm{DE}$ - Differentially Expressed; $\boldsymbol{\mu G}$ Microgravity

\section{Declarations}

\section{Ethics approval and consent to participate}

All subjects were informed about the experimental procedures and gave their written consent. The experimental protocol conformed to the standards set by the Declaration of Helsinki and was approved 
by the local Ethic Committee (CPP Sud-Ouest Outre-Mer I, France) and French Health Authorities $\left(\boldsymbol{n}^{\circ} I D\right.$ RCB: 2014-A 00904-43).

\section{Consent for publication}

The authors affirm that human research participants provided informed consent for data collected being used for publication, and a number of publications from this DI study protocol have since been published. All authors have read and approved the final manuscript for publication.

\section{Availability of data and material}

The datasets generated during and/or analysed during the current study are available from the corresponding author on reasonable request.

\section{Competing interests}

All other authors report no financial, professional or personal conflicts of interest relating to this publication.

\section{Funding}

This dry immersion protocol was supported by CNES (DAR CNES Nº $2018-4800000970)$. R.P.M. acknowledges the funding support of Science Foundation Ireland (11/TIDA/B1927), Health Research Board of Ireland (HRA/2009/122), Enterprise Ireland and the European Regional Development Fund (ERDF) under Ireland's European Structural and Investment Funds programmes (PC-2009-0311, INV/13000/001/2015, IP 2015 0399Y, IP 20150418 \& IP 2017 0587). L.T. was in part supported by an Irish Research Council for Science, Engineering \& Technology (IRCSET) EMBARK Postgraduate Scholarship 2012-2015 (RS/2012/2499). RPM is a management committee member of the EU H2020 COST actions- CM1406 \& CA18127.

\section{Authors contributions}

All authors approved the final version to be published. Conceptualization: Nastassia Navasiolava, MariePierre Bareille, Guillemette Gauquelin-Koch, Françoise Larcher, Gerardene Meade-Murphy, Claude Gharib, Marc-Antoine Custaud, Ronan P. Murphy; Data curation: Laura Twomey, Nastassia Navasiolava, Adrien Robin, Marie-Pierre Bareille, Guillemette Gauquelin-Koch, Arnaud Beck, Françoise Larcher, Patricia B. Maguire, Marc-Antoine Custaud, Ronan P. Murphy; Formal analysis: Laura Twomey, Nastassia Navasiolava, Adrien Robin, Marie-Pierre Bareille, Guillemette Gauquelin-Koch, Arnaud Beck, Françoise Larcher, Gerardene Meade-Murphy, Sinead Sheridan, Patricia B. Maguire, Michael Harrison, Bernard Degryse, Niall M. Moyna, Claude Gharib, Marc-Antoine Custaud, Ronan P. Murphy; Funding acquisition: Laura Twomey, Nastassia Navasiolava, Françoise Larcher, Claude Gharib, Marc-Antoine Custaud, Ronan P. Murphy; Investigation: Laura Twomey, Nastassia Navasiolava, Adrien Robin, MariePierre Bareille, Guillemette Gauquelin-Koch, Arnaud Beck, Françoise Larcher, Patricia B. Maguire, MarcAntoine Custaud, Ronan P. Murphy; Methodology: Laura Twomey, Nastassia Navasiolava, Adrien Robin, Marie-Pierre Bareille, Guillemette Gauquelin-Koch, Arnaud Beck, Françoise Larcher, Gerardene Meade- 
Murphy, Sinead Sheridan, Patricia B. Maguire, Michael Harrison, Bernard Degryse, Niall M. Moyna, Claude Gharib, Marc-Antoine Custaud, Ronan P. Murphy; Project administration: Nastassia Navasiolava, MariePierre Bareille, Guillemette Gauquelin-Koch, Arnaud Beck, Françoise Larcher, Claude Gharib, Marc-Antoine Custaud, Ronan P. Murphy; Resources: Marie-Pierre Bareille, Guillemette Gauquelin-Koch, Arnaud Beck, Françoise Larcher, Patricia B. Maguire, Claude Gharib, Marc-Antoine Custaud, Ronan P.

Murphy; Supervision: Nastassia Navasiolava, Marie-Pierre Bareille, Guillemette Gauquelin-Koch, Arnaud Beck, Françoise Larcher, Patricia B. Maguire, Claude Gharib, Marc-Antoine Custaud, Ronan P. Murphy; Validation:Laura Twomey, Nastassia Navasiolava, Adrien Robin, Marie-Pierre Bareille, Guillemette Gauquelin-Koch, Arnaud Beck, Françoise Larcher, Gerardene Meade-Murphy, Sinead Sheridan, Patricia B. Maguire, Michael Harrison, Bernard Degryse, Niall M. Moyna, Claude Gharib, Marc-Antoine Custaud, Ronan P. Murphy; Visualization: Nastassia Navasiolava, Adrien Robin, Marc-Antoine Custaud, Ronan P. Murphy; Writing - original draft: Laura Twomey, Nastassia Navasiolava, Adrien Robin, MarcAntoine Custaud, Ronan P. Murphy; Writing - review \& editing: All authors.

\section{Acknowledgements}

We thank the volunteers and the staff of MEDES (Toulouse, France) for their participation in this study.

\section{Authors' information}

Laura Twomey ${ }^{1,2 *}$, Nastassia Navasiolava ${ }^{3 *}$, Adrien Robin ${ }^{3}$, Marie-Pierre Bareille ${ }^{4}$, Guillemette GauquelinKoch $^{5}$, Arnaud Beck ${ }^{4}$, Françoise Larcher ${ }^{6}$, Gerardene Meade-Murphy ${ }^{7}$, Sinead Sheridan ${ }^{8}$, Patricia B. Maguire $^{9}$, Michael Harrison ${ }^{10}$, Bernard Degryse ${ }^{2,12}$, Niall M. Moyna ${ }^{11,12}$, Claude Gharib ${ }^{13}$, Marc-Antoine Custaud $^{3}$, Ronan P. Murphy ${ }^{2,12}$

1 Technological University Dublin, Ireland

${ }^{2}$ Cell \& Molecular Physiology Group, School of Health \& Human Performance, Dublin City University, Glasnevin, Dublin 9, Ireland

3 Univ Angers, CHU Angers, CRC, Inserm, CNRS, MITOVASC, SFR ICAT, F-49000 Angers, France

${ }^{4}$ MEDES, Toulouse, France

${ }^{5}$ Centre National d'Études Spatiales (CNES), Paris, France

${ }^{6} \mathrm{CHU}$ Angers, Laboratoire de Biochimie, Univ Angers, F-49000 Angers, France

7 Department of Pharmacology and Therapeutics, University College Cork, Cork, Ireland

8 Department of Sports Science \& Physical Education, The Chinese University of Hong Kong

${ }^{9}$ Conway-SPHERE Research Group, Conway Institute, University College Dublin, Dublin, Ireland 
${ }^{10}$ Department of Sport and Exercise Science, Waterford Institute of Technology, Cork Road, Waterford, Ireland

11 Vascular Physiology and Clinical Exercise Medicine Group, School of Health \& Human Performance, DCU, Glasnevin, D9, Ireland

12 Centre for Preventive Medicine, DCU, Glasnevin, D9, Ireland

${ }^{13}$ Institut NeuroMyoGène, Faculté de Médecine Lyon-Est, Université de Lyon, Lyon, France

\section{References}

1. Bhatnagar, A. Environmental Determinants of Cardiovascular Disease. Circ Res, 121 (2), 162-180 (2017).

2. Fiuza-Luces, C. et al. Exercise benefits in cardiovascular disease: beyond attenuation of traditional risk factors. Nat Rev Cardiol, 15 (12), 731-743 (2018).

3. Giladi, A. et al. Single-cell characterization of haematopoietic progenitors and their trajectories in homeostasis and perturbed haematopoiesis. Nat Cell Biol, 20 (7), 836-846 (2018).

4. Sanjuan-Pla, A. et al. Platelet-biased stem cells reside at the apex of the haematopoietic stem-cell hierarchy. Nature, 502 (7470), 232-236 (2013).

5. Yang, J. et al. Epigenetic regulation of megakaryocytic and erythroid differentiation by PHF2 histone demethylase. J Cell Physiol, 233 (9), 6841-6852 (2018).

6. Shen, B. et al. A mechanosensitive peri-arteriolar niche for osteogenesis and lymphopoiesis. Nature, 591 (7850), 438-444 (2021).

7. Elgheznawy, A. \& Fleming, I. Platelet-Enriched MicroRNAs and Cardiovascular Homeostasis. Antioxid Redox Signal, 29 (9), 902-921 (2018).

8. Sunderland, N. et al. MicroRNA Biomarkers and Platelet Reactivity: The Clot Thickens. Circ Res, 120 (2), 418-435 (2017).

9. Wallace, R. G. et al. The role of epigenetics in cardiovascular health and ageing: A focus on physical activity and nutrition. Mech Ageing Dev, 174, 76-85 (2018).

10. Izzi, B. et al. Variation of PEAR1 DNA methylation influences platelet and leukocyte function. Clin Epigenetics, 11 (1), 151 (2019).

11. Indumathi, B. et al. Probing the epigenetic signatures in subjects with coronary artery disease. $\mathrm{Mol}$ Biol Rep, 47 (9), 6693-6703 (2020).

12. Gao, S. et al. Epigenetic modulation of glycoprotein VI gene expression by DNA methylation. Life Sci, 241, 117103 (2020). 
13. Su, J. et al. Association of P2Y12 gene promoter DNA methylation with the risk of clopidogrel resistance in coronary artery disease patients. Biomed Res Int, 2014, 450814 (2014).

14. Aslan, J. E. Platelet Proteomes, Pathways, and Phenotypes as Informants of Vascular Wellness and Disease. Arterioscler Thromb Vasc Biol, 41 (3), 999-1011 (2021).

15. Hindle, M. S. et al. Multidimensional flow cytometry reveals novel platelet subpopulations in response to prostacyclin (J Thromb Haemost, 2021).

16. Izzi, B. et al. Learning by counting blood platelets in population studies: survey and perspective a long way after Bizzozero. J Thromb Haemost, 16 (9), 1711-1721 (2018).

17. Widlansky, M. E. The danger of sedenterism: endothelium at risk. Am J Physiol Heart Circ Physiol, 299 (2), 243-244 (2010).

18. Nosova, E. V. et al. Short-term physical inactivity impairs vascular function. J Surg Res, 190 (2), $672-$ 682 (2014).

19. Hughson, R. L. Recent findings in cardiovascular physiology with space travel. Respir Physiol Neurobiol, 169 (Suppl 1), 38-41 (2009).

20. Navasiolava, N. M. et al. Long-term dry immersion: review and prospects. Eur J Appl Physiol, 111 (7), 1235-1260 (2011).

21. Navasiolava, N. M. et al. Skin vascular resistance in the standing position increases significantly after 7 days of dry immersion. Auton Neurosci, 160 (1-2), 64-68 (2011).

22. Navasiolava, N. M. et al. Enforced physical inactivity increases endothelial microparticle levels in healthy volunteers. Am J Physiol Heart Circ Physiol, 299 (2), 248-256 (2010).

23. Navasiolava, N. M. et al. NT-ProBNP levels, water and sodium homeostasis in healthy men: effects of 7 days of dry immersion. Eur J Appl Physiol, 111 (9), 2229-2237 (2011).

24. Coupé, M. et al. Cardiovascular deconditioning: From autonomic nervous system to microvascular dysfunctions. Respir Physiol Neurobiol, 169 (Suppl 1), 10-12 (2009).

25. Kermorgant, M. et al. Impacts of Simulated Weightlessness by Dry Immersion on Optic Nerve Sheath Diameter and Cerebral Autoregulation. Front Physiol, 8, 780 (2017).

26. Pöyhönen, T. \& Avela, J. Effect of head-out water immersion on neuromuscular function of the plantarflexor muscles. Aviat. Space Environ. Med, 73 (12), 1215-1218 (2002).

27. Linossier, M. T. et al. Effects of short-term dry immersion on bone remodeling markers, insulin and adipokines. PLoS One, 12 (8), 0182970 (2017).

28. Tomilovskaya, E. et al. Dry Immersion as a Ground-Based Model of Microgravity Physiological Effects. Front Physiol, 10, 284 (2019).

29. De Abreu, S. et al. Multi-System Deconditioning in 3-Day Dry Immersion without Daily Raise. Front Physiol, 8, 799 (2017).

30. Demangel, R. et al. Early structural and functional signature of 3-day human skeletal muscle disuse using the dry immersion model. J Physiol, 595 (13), 4301-4315 (2017). 
31. Treffel, L. et al. DI-5-Cuffs: Lumbar Intervertebral Disc Proteoglycan and Water Content Changes in Humans after Five Days of Dry Immersion to Simulate Microgravity.Int J Mol Sci, 2020. 21(11).

32. Ogoh, S. et al. Internal carotid, external carotid and vertebral artery blood flow responses to 3 days of head-out dry immersion. Exp Physiol, 102 (10), 1278-1287 (2017).

33. Acket, B. et al. Dry immersion as a model of deafferentation: A neurophysiology study using somatosensory evoked potentials. PLoS One, 13 (8), 0201704 (2018).

34. Senis, Y. A. et al. A comprehensive proteomics and genomics analysis reveals novel transmembrane proteins in human platelets and mouse megakaryocytes including $\mathrm{G} 6 \mathrm{~b}-\mathrm{B}$, a novel immunoreceptor tyrosine-based inhibitory motif protein. Mol Cell Proteomics, 6 (3), 548-564 (2007).

35. Schwarz, U. R. et al. Flow cytometry analysis of intracellular VASP phosphorylation for the assessment of activating and inhibitory signal transduction pathways in human platelets-definition and detection of ticlopidine/clopidogrel effects. Thromb Haemost, 82 (3), 1145-1152 (1999).

36. Grigor'ev, A. I., Kozlovskaia, I. B. \& Shenkman, B. S. [The role of support afferents in organisation of the tonic muscle system] 90p. 508-21(Ross Fiziol Zh Im I M, 2004). Sechenova5

37. Kirichenko, L. L. et al. [Parameters of hemostasis in persons with neurocirculatory dystonia under conditions of "dry" immersion]. Kosm Biol Aviakosm Med, 22 (1), 10-13 (1988).

38. Kirichenko, L. L., Smirnov, V. V. \& Evdokimova, A. G. [State of microcirculation and cellular hemostasis in men with borderline arterial hypertension during thermoneutral "dry" water immersion]. Kosm Biol Aviakosm Med, 19 (5), 35-38 (1985).

39. Mangala, L. S. et al. Effects of simulated microgravity on expression profile of microRNA in human lymphoblastoid cells. J Biol Chem, 286 (37), 32483-32490 (2011).

40. Girardi, C. et al. Integration analysis of microRNA and mRNA expression profiles in human peripheral blood lymphocytes cultured in modeled microgravity. Biomed Res Int, 2014, 296747 (2014).

41. Malkani, S. et al. Circulating miRNA Spaceflight Signature Reveals Targets for Countermeasure Development. Cell Rep, 33 (10), 108448 (2020).

42. Larina, I. M. et al. [Seven-day dry immersion: relations between water-electrolyte and cardiovascular responses]. Fiziol Cheloveka, 37 (5), 100-107 (2011).

43. Watenpaugh, D. E. et al. Effects of spaceflight on human calf hemodynamics. J Appl Physiol (1985), 90 (4), 1552-1558 (2001).

44. Cao, D. et al. Hematopoietic stem cells and lineage cells undergo dynamic alterations under microgravity and recovery conditions. Faseb j, 33 (6), 6904-6918 (2019).

45. Ivanova, S. M. et al. [Investigation of the morphofunctional properties of human red blood cells under the conditions of 7-day dry immersion]. Aviakosm Ekolog Med, 43 (1), 43-47 (2009).

46. Ivanova, S. M. et al. [Examination of the morphofunctional properties of red blood cells of humans in 5-day dry immersioin]. Aviakosm Ekolog Med, 45 (6), 49-51 (2011).

47. Berendeeva, T. A. et al. [Human immunity system status during 7-day dry immersion]. Aviakosm Ekolog Med, 43 (5), 36-42 (2009). 
48. Watenpaugh, D. E. Fluid volume control during short-term space flight and implications for human performance. J Exp Biol, 204 (Pt 18), 3209-3215 (2001).

49. Yin, X. et al. Interplay of Proximal Flow Confluence and Distal Flow Divergence in Patient-Specific Vertebrobasilar System. PLoS One, 11 (7), 0159836 (2016).

50. Ivanov, A. P. et al. [Changes in several indicators of blood rheology in experiments simulating weightlessness]. Kosm Biol Aviakosm Med, 17 (6), 25-30 (1983).

51. Kim, B. J. et al. A physiometer for simultaneous measurement of whole blood viscosity and its determinants: hematocrit and red blood cell deformability. Analyst, 144 (9), 3144-3157 (2019).

52. Kuzichkin, D. S. et al. [Hemostasis system indices after short-term space flights and during 7-day "dry" immersion experiment]. Fiziol Cheloveka, 36 (4), 125-129 (2010).

53. Vij, A. G. Effect of prolonged stay at high altitude on platelet aggregation and fibrinogen levels., 20 (6), 421-427 (2009).

54. Schumacher, W. A. et al. Biomarker optimization to track the antithrombotic and hemostatic effects of clopidogrel in rats. J Pharmacol Exp Ther, 322 (1), 369-377 (2007).

55. Gaglia, M. A. et al. Correlation between light transmission aggregometry, VerifyNow P2Y12, and VASP-P platelet reactivity assays following percutaneous coronary intervention. J Interv Cardiol, 24 (6), 529-534 (2011).

56. Assinger, A., Schmid, W. \& Volf, I. Decreased VASP phosphorylation in platelets of male and female smokers of young age., 21 (8), 596-603 (2010).

57. Wang, X. et al. Circulating heat shock protein 27 as a novel marker of subclinical atherosclerosis in type 2 diabetes: a cross-sectional community-based study. BMC Cardiovasc Disord, 20 (1), 198 (2020).

58. Szyller, J. \& Bil-Lula, I. Heat Shock Proteins in Oxidative Stress and Ischemia/Reperfusion Injury and Benefits from Physical Exercises: A Review to the Current Knowledge. Oxid Med Cell Longev 2021. 2021: p. 6678457.

59. Tokuda, H. et al. Release of Phosphorylated HSP27 (HSPB1) from Platelets Is Accompanied with the Acceleration of Aggregation in Diabetic Patients. PLoS One, 10 (6), 0128977 (2015).

60. Enomoto, Y. et al. cAMP regulates ADP-induced HSP27 phosphorylation in human platelets. Int J Mol Med, 27 (5), 695-700 (2011).

61. Kattoor, A. J., Kanuri, S. H. \& Mehta, J. L. Role of Ox-LDL and LOX-1 in Atherogenesis. Curr Med Chem, 26 (9), 1693-1700 (2019).

62. Marwali, M. R. et al. Modulation of ADP-induced platelet activation by aspirin and pravastatin: role of lectin-like oxidized low-density lipoprotein receptor-1, nitric oxide, oxidative stress, and inside-out integrin signaling. J Pharmacol Exp Ther, 322 (3), 1324-1332 (2007).

63. Yan, M., Mehta, J. L. \& Hu, C. LOX-1 and obesity. Cardiovasc Drugs Ther, 25 (5), 469-476 (2011).

64. Ueland, T. et al. Dickkopf-1 enhances inflammatory interaction between platelets and endothelial cells and shows increased expression in atherosclerosis. Arterioscler Thromb Vasc Biol, 29 (8), 
1228-1234 (2009).

65. Pronobis, M. I., Rusan, N. M. \& Peifer, M. A novel GSK3-regulated APC:Axin interaction regulates Wnt signaling by driving a catalytic cycle of efficient $\beta$ catenin destruction. Elife, 4, 08022 (2015).

66. Macaulay, I. C. et al. Canonical Wnt signaling in megakaryocytes regulates proplatelet formation., 121 (1), 188-196 (2013).

67. Steele, B. M. et al. Canonical Wnt signaling negatively regulates platelet function. Proc Natl Acad Sci US A, 106 (47), 19836-19841 (2009).

68. Steele, B. M. et al. WNT-3a modulates platelet function by regulating small GTPase activity. FEBS Lett, 586 (16), 2267-2272 (2012).

69. Henson, J. et al. Sedentary time and markers of chronic low-grade inflammation in a high risk population. PLoS One, 8 (10), 78350 (2013).

70. Bester, J. \& Pretorius, E. Effects of IL-1 $\beta$, IL- 6 and IL-8 on erythrocytes, platelets and clot viscoelasticity. Sci Rep, 6, 32188 (2016).

71. Ishibashi, T. et al. Human interleukin 6 is a direct promoter of maturation of megakaryocytes in vitro. Proc Natl Acad Sci U S A, 86 (15), 5953-5957 (1989).

72. Plé, H. et al. The repertoire and features of human platelet microRNAs. PLoS One, 7 (12), 50746 (2012).

73. Teruel-Montoya, R. et al. MicroRNA expression differences in human hematopoietic cell lineages enable regulated transgene expression. PLoS One, 9 (7), 102259 (2014).

74. Edelstein, L. C. et al. MicroRNAs in platelet production and activation. J Thromb Haemost, 2013. 11 Suppl 1: p. $340-50$.

75. Cai, J. et al. MicroRNA-374a activates Wnt/ $\beta$-catenin signaling to promote breast cancer metastasis. J Clin Invest, 123 (2), 566-579 (2013).

76. Kim, S. Y. et al. Wnt5a potentiates U46619-induced platelet aggregation via the PI3K/Akt pathway. Mol Cells, 32 (4), 333-336 (2011).

77. Koyama-Nasu, R. et al. Thr160 of Axin 1 is critical for the formation and function of the $\beta$-catenin destruction complex. Biochem Biophys Res Commun, 459 (3), 411-415 (2015).

78. Domańska-Senderowska, D. et al. MicroRNA Profile and Adaptive Response to Exercise Training: A Review. Int J Sports Med, 40 (4), 227-235 (2019).

79. Hibler, E. et al. Impact of a diet and activity health promotion intervention on regional patterns of DNA methylation. Clin Epigenetics, 11 (1), 133 (2019).

80. Meder, B. et al. MicroRNA signatures in total peripheral blood as novel biomarkers for acute myocardial infarction. Basic Res Cardiol, 106 (1), 13-23 (2011).

\section{Highlights}

\section{Title Page}




\section{Highlights}

- Dry Immersion, a ground based model of microgravity, elicits a prothrombotic platelet phenotype and altered platelet indices

- miRNA signature of platelets is altered by Dry Immersion

- Altered Cardiovascular disease and Inflammation biomarker signature of microgravity (3-day Dry Immersion) observed in test subjects

- Correlation between miRomic profile and observed biomarker levels

- DKK1 and Axin-1 levels implicate Wnt2a signalling in physical inactivity/microgravity-induced platelet phenotype

\section{Basic Research}

- Platelets

- Translational Studies

- Physiology

- Vascular Biology

\section{Epidemiology, Lifestyle, and Prevention}

- Cardiovascular Disease

- Lifestyle

\section{Genetics}

- Epigenetics

\section{Vascular Disease}

Thrombosis

\section{Figures}




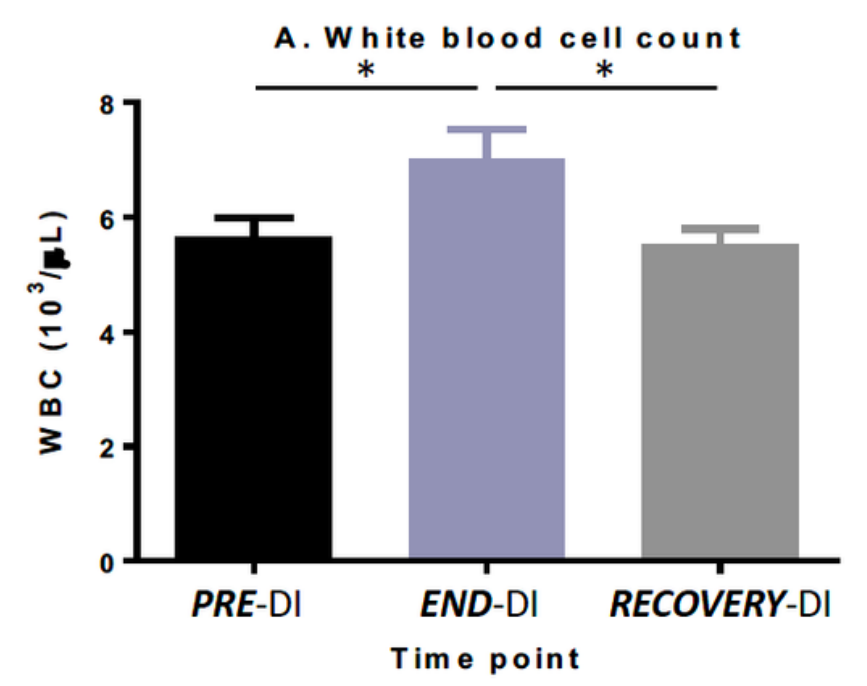

C. Hemoglobin

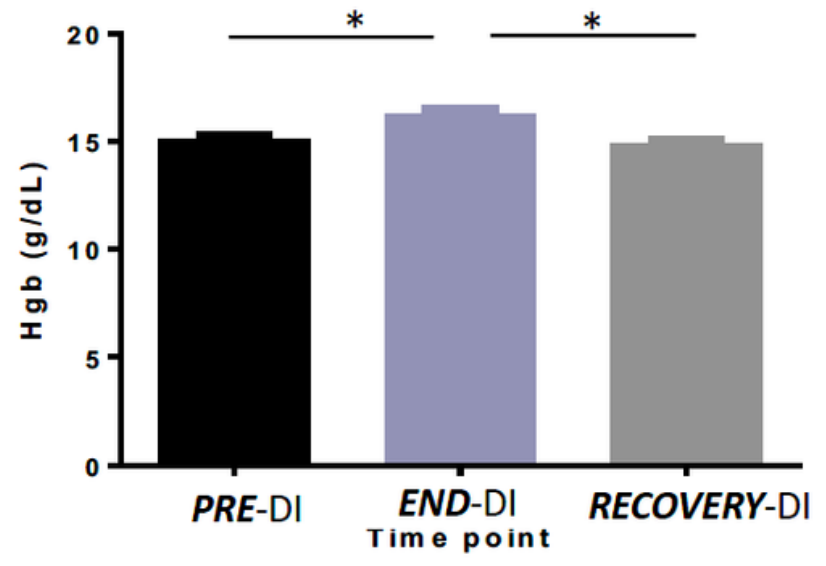

B. Red blood cell count

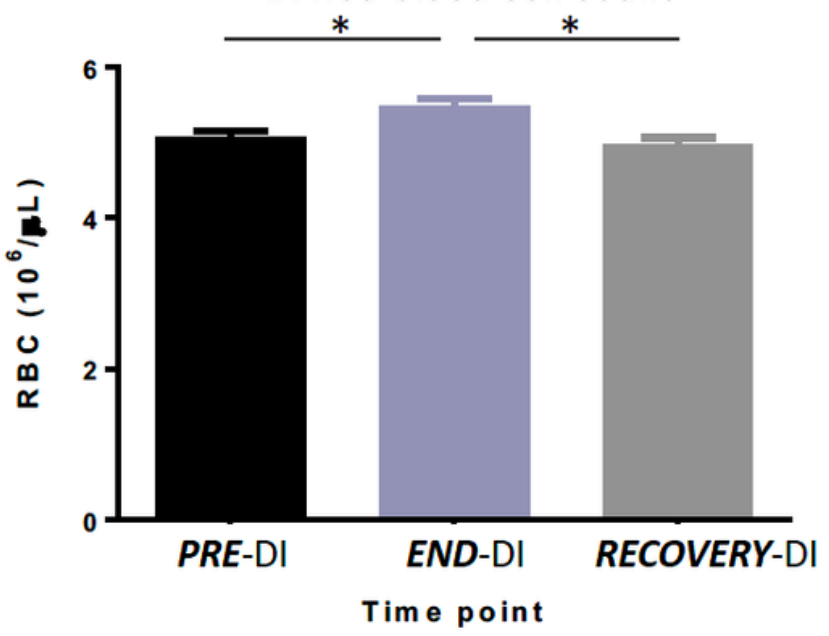

D. Hem a tocrit

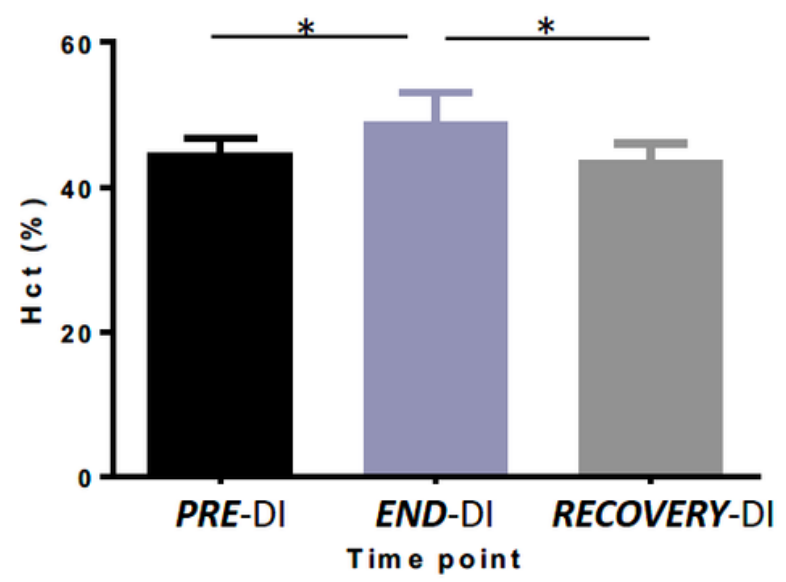

Figure 1

Effect of dry immersion and physical inactivity on RBC and WBC characteristics. Graphs represent the mean \pm SEM of each parameter at each time point. (A) White blood cell count, (B) Red blood cell count, (C) Haemoglobin and (D) Haematocrit. ${ }^{*} P<0.05$. Paired samples t-test and repeated measures ANOVA (adjusted for age, BMI and V02; $\mathrm{n}=12$, biological replicates). 

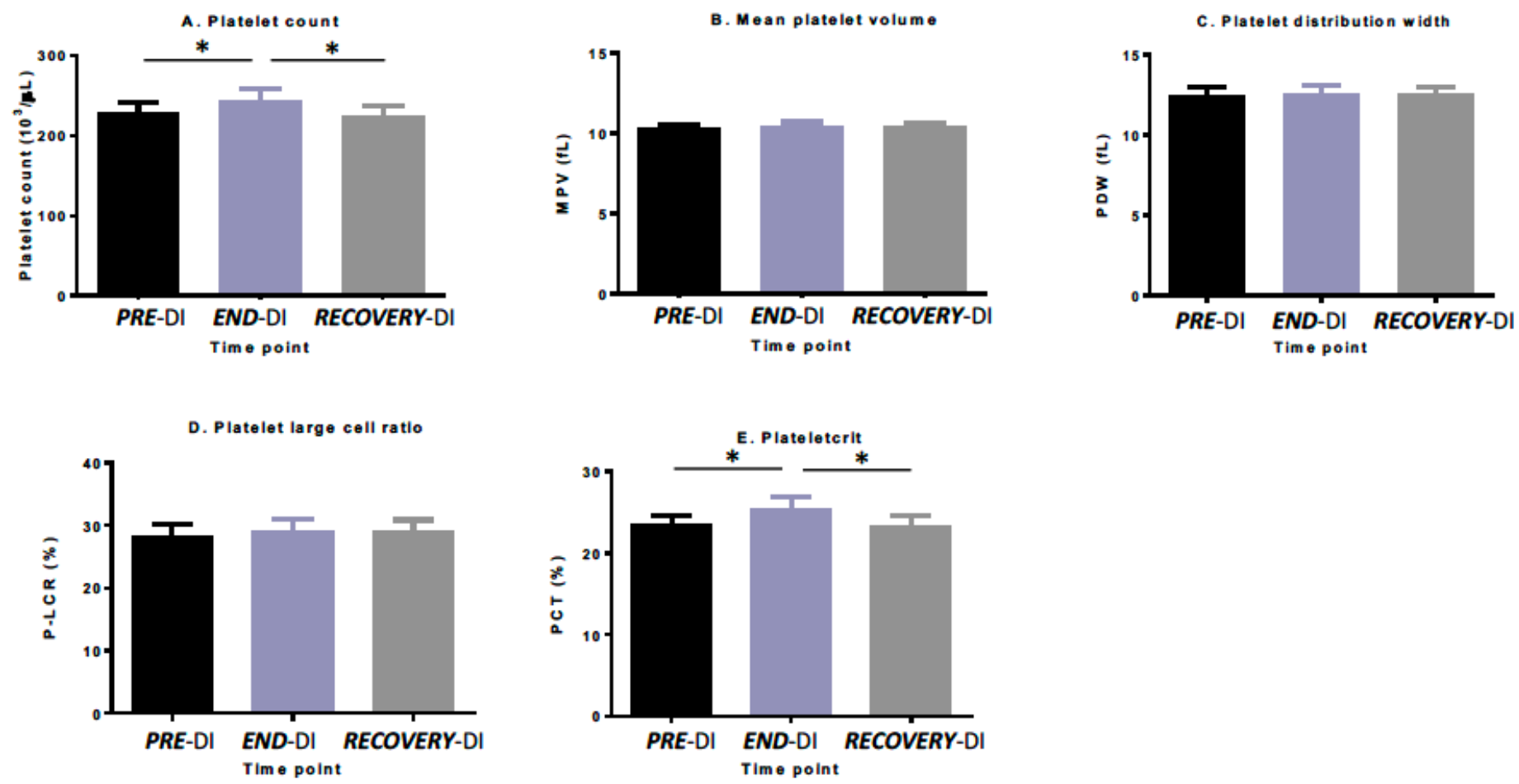

\section{Figure 2}

Effect of dry immersion and physical inactivity on platelet indices. Graphs represent the mean \pm SEM of each parameter at each time point. (A) Platelet count, (B) Mean platelet volume, (C) Platelet distribution width, (D) Platelet large cell ratio and (E) Plateletcrit. * $P<0.05$. Paired samples t-test and repeated measures ANOVA (adjusted for age, BMI and VO2; $n=12$, biological replicates). 
A. A card upregulated miRnA

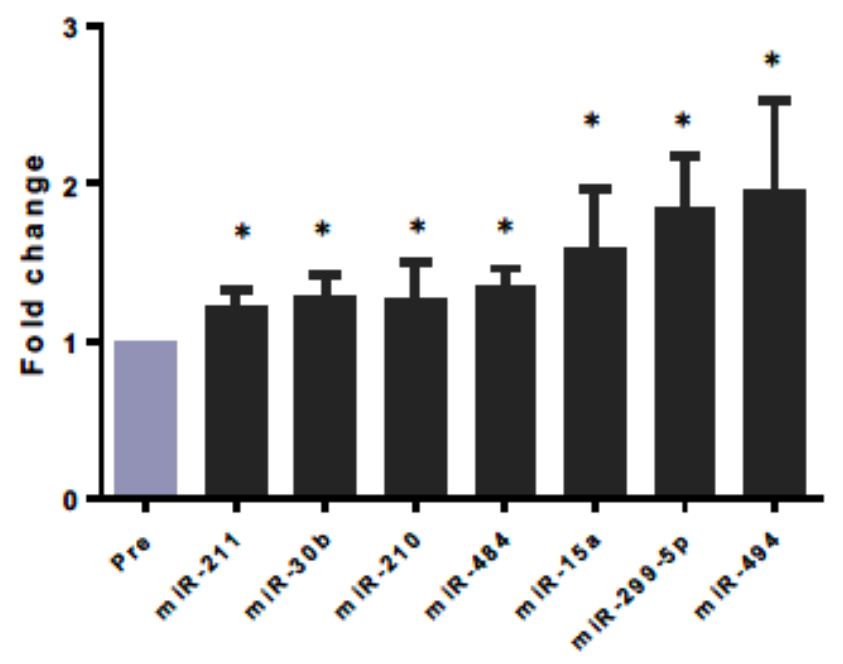

microR NA
B. A card downregulated miRNA

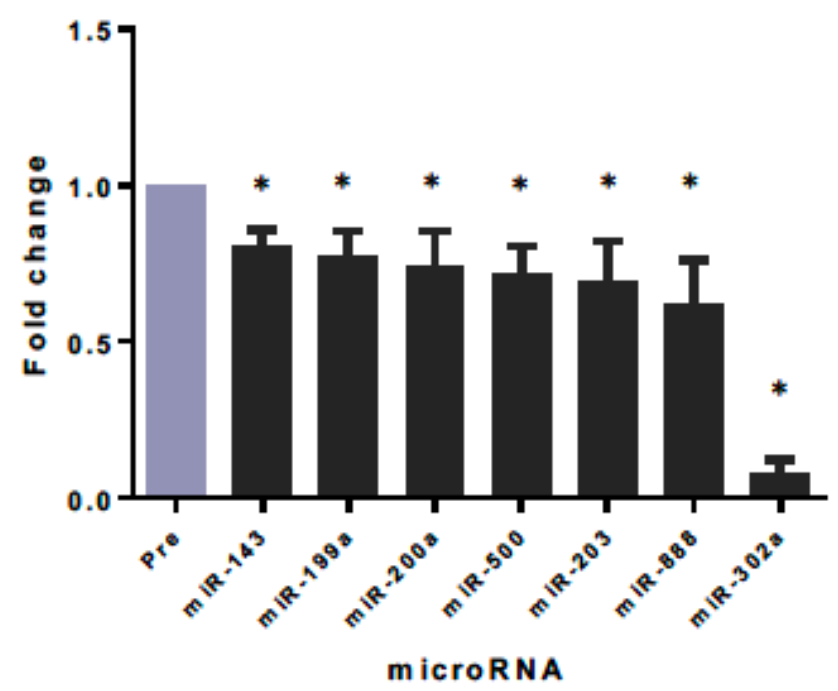

D. B card down regulated miRnA

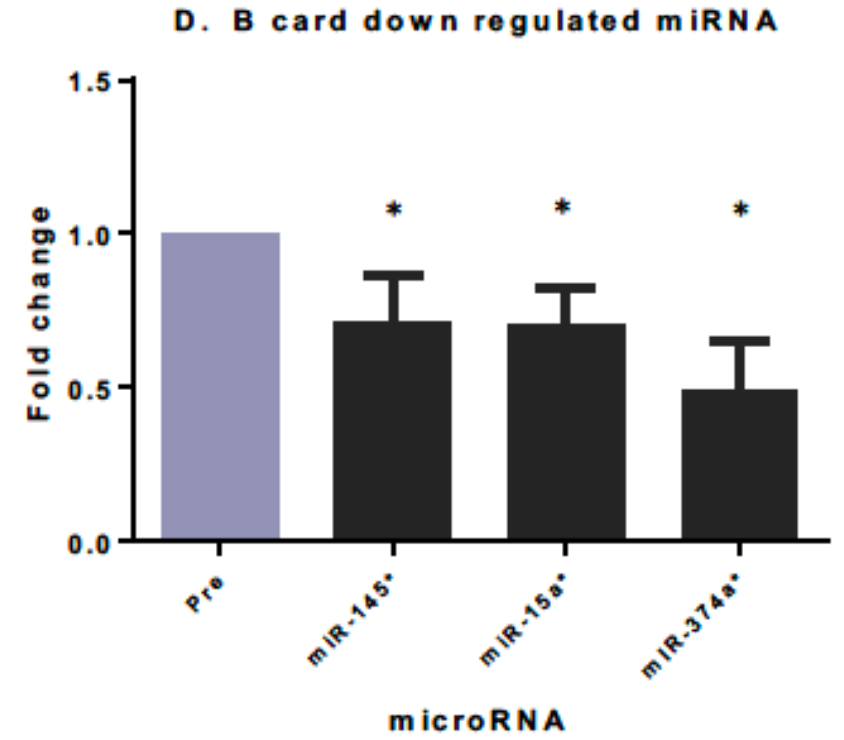

microR N A

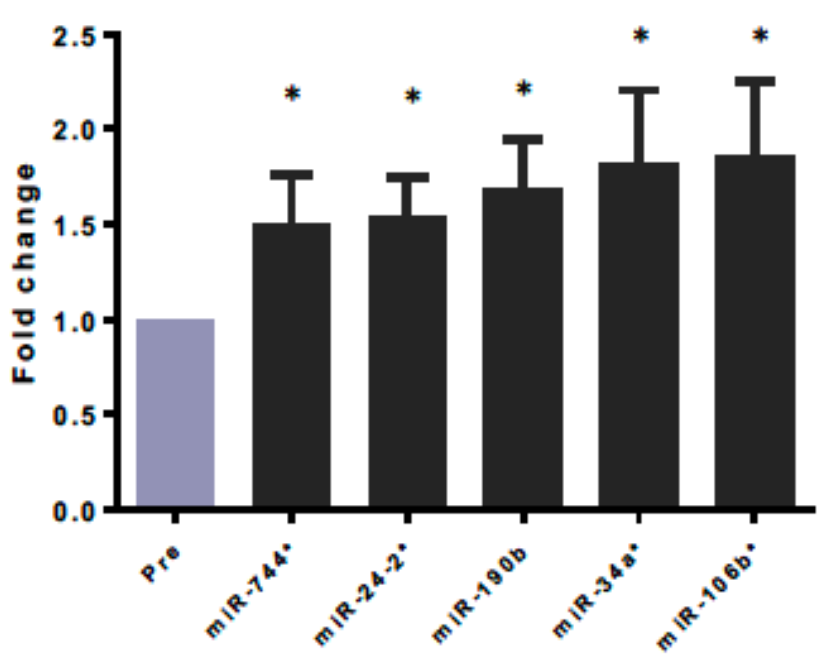

m icroR NA

Figure 3

(A) Effect of dry immersion on platelet function assessed by Impact R analysis. Graphs represent the mean \pm SEM of each parameter at each time point. (i) Platelet Adhesion and (ii) Platelet Aggregation. * $P<0.05$. Paired samples t-test and repeated measures ANOVA (adjusted for age, BMI and VO2; $n=12$ biological replicates, technical replicates $n=3$ for each subject). (B) Impact $R$ images from subject $J$ at each stage of dry immersion. Image shows platelet adhesion and aggregation at (i) PRE-DI, (ii) END-DI and (iii) RECOVERY-DI. 
A

(i) Platelet Adhesion

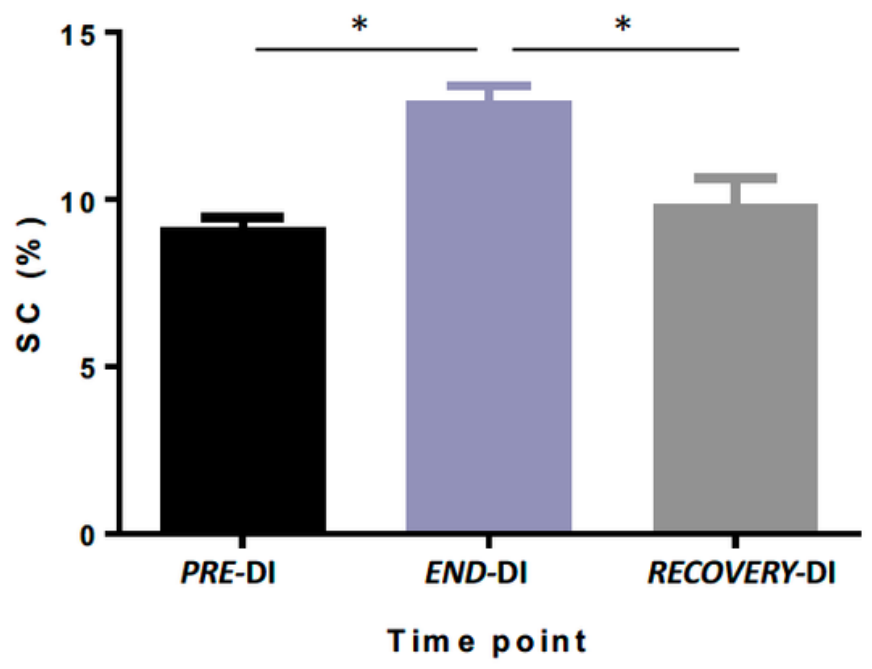

(ii) Platelet Aggregation

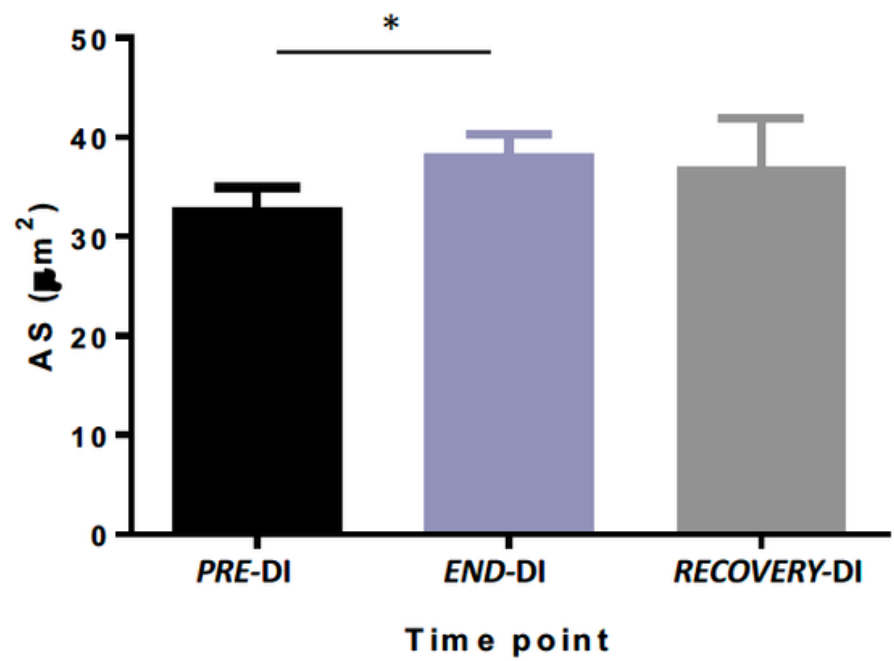

(iii) RECOVERY-DI

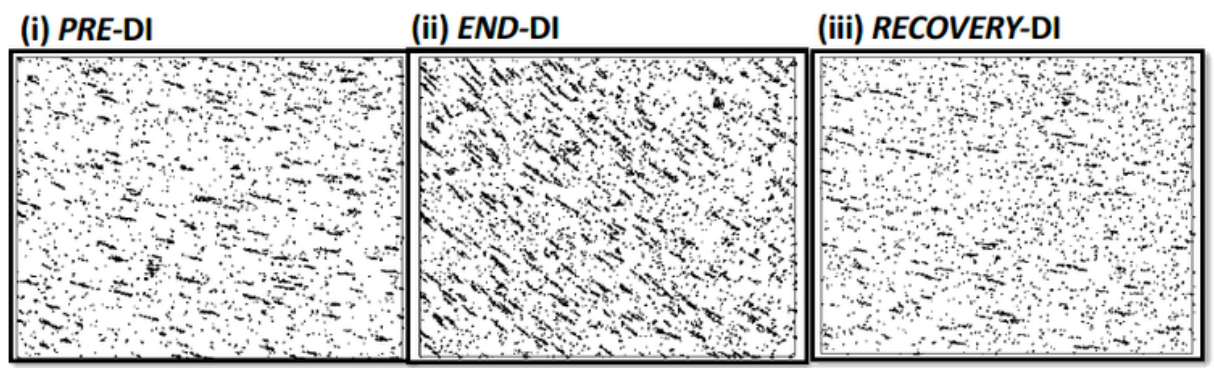

\section{Figure 4}

Effect of dry immersion on plasma cardiovascular protein biomarkers. The graphs represent mean \pm SEM. (A) Heat shock protein 27, (B) Lectin like oxidised LDL receptor, (C) NF KappaB essential modulator, (D) Proto-oncogene tyrosine protein kinase and (E) Dickkopf related protein. * $\mathrm{P}<0.05$, Paired samples ttest and repeated measures ANOVA (adjusted for age, BMI and VO2; $\mathrm{n}=12$ biological replicates). 
A. Heat shock protein 27

(HS P -27)

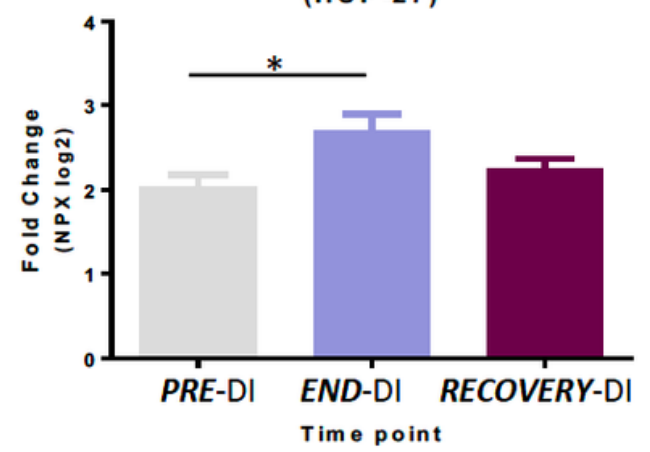

D. Proto-oncogene tyrosine-protein kinase

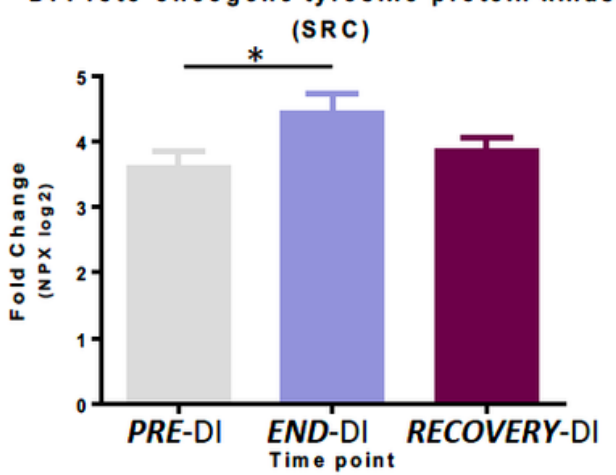

B. Lectin-like oxidized LDL receptor (LOX-1)

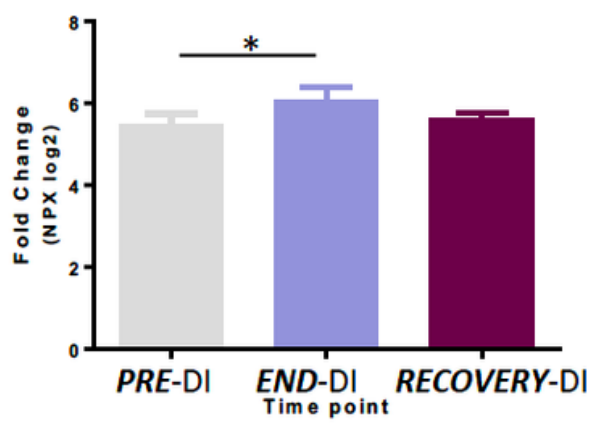

E. Dickkopf-related protein

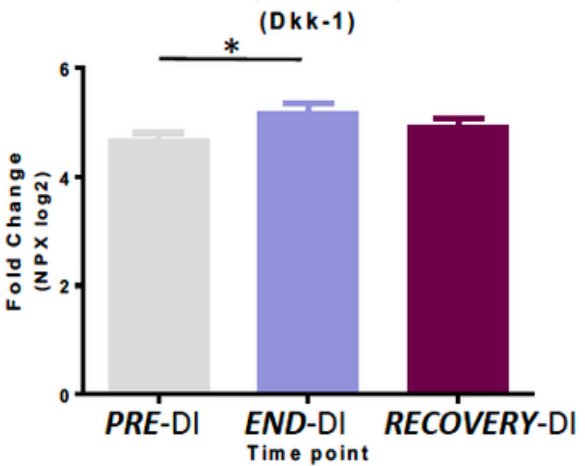

C. NF-kappa-B essential modulator (NEMO)

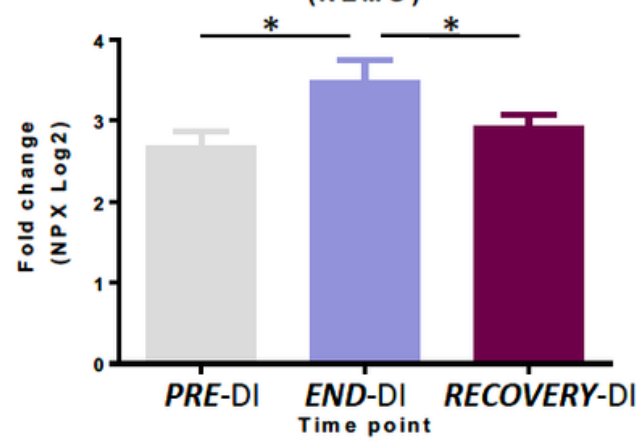

\section{Figure 5}

Effect of dry immersion on inflammatory protein biomarkers. All data are expressed mean \pm SEM. (A) Axin1, (B) SIRT2, (C) Interleukin-6, (D) Matrix metalloproteinase-3 and (E) Matrix metalloproteinase 10. Axin1 and MMP-3 were specific to INF panel while II-6, SIRT2 and MMP-10 were also found on the CVD panel. * $P<0.05$, Paired samples t-test and repeated measures ANOVA (adjusted for age, BMI and VO2; $\mathrm{n}=12$ biological replicates). 


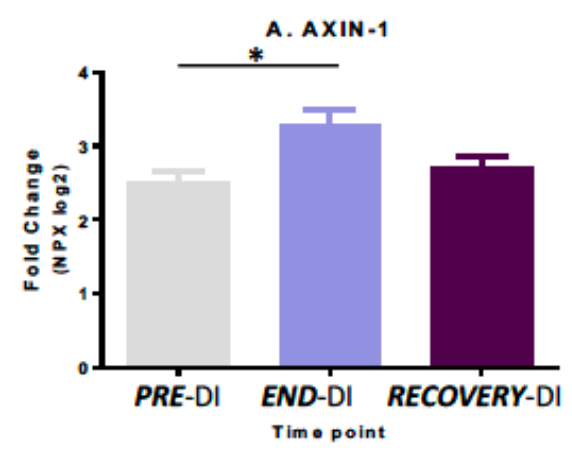

D. Matrix $m$ etalloproteinase 3 (M M P - 3)

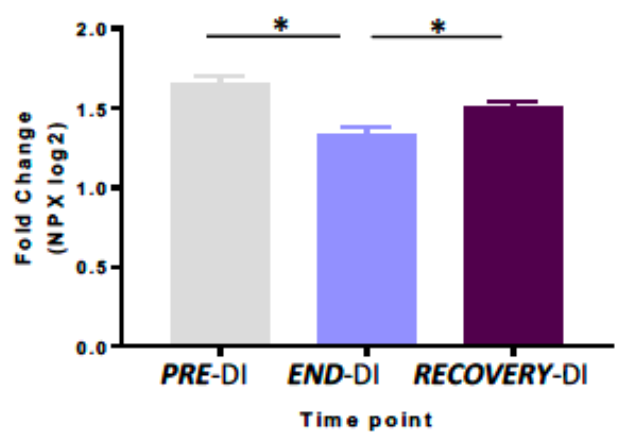

B. SIRT2

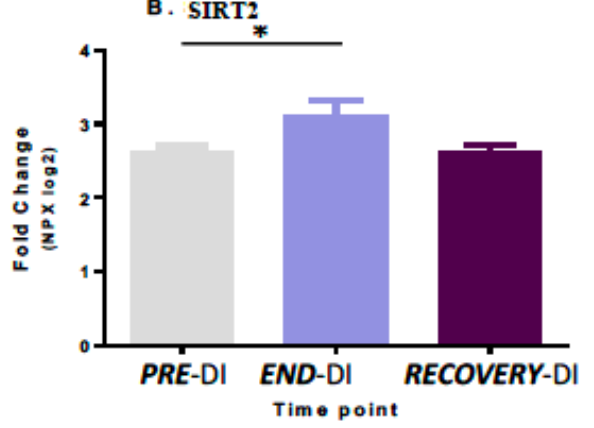

E. Matrix metalloproteinase 10 (M M P - 10)

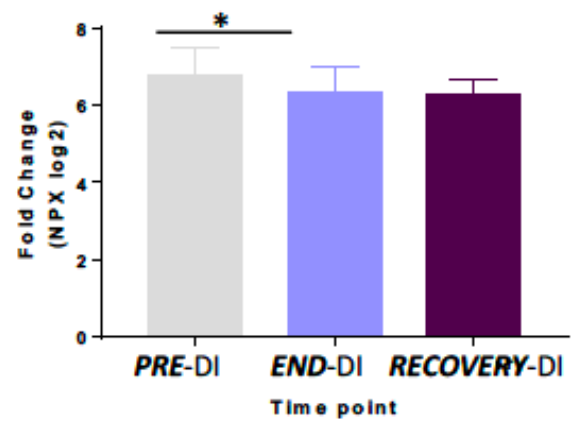

Figure 6

Differentially expressed miRNA at the post dry immersion time point. miRNA profiles were assessed only at the pre-DI and post-DI time points. This figure shows miRNA which were differentially expressed at the post-DI compared to pre-DI time point. All data are expressed mean \pm SEM. Graph A shows miRNA that were up regulated after $D I$ on the $A$ card, and graph $B$ shows miRNA that were downregulated after DI on the A card. Graph $C$ shows B card up regulated miRNA, whilst graph D shows B card downregulated miRNA. * $P<0.05$, Paired samples t-test and repeated measures ANOVA, $n=8$ biological replicates. 
A. Down regulated targets of DKK1

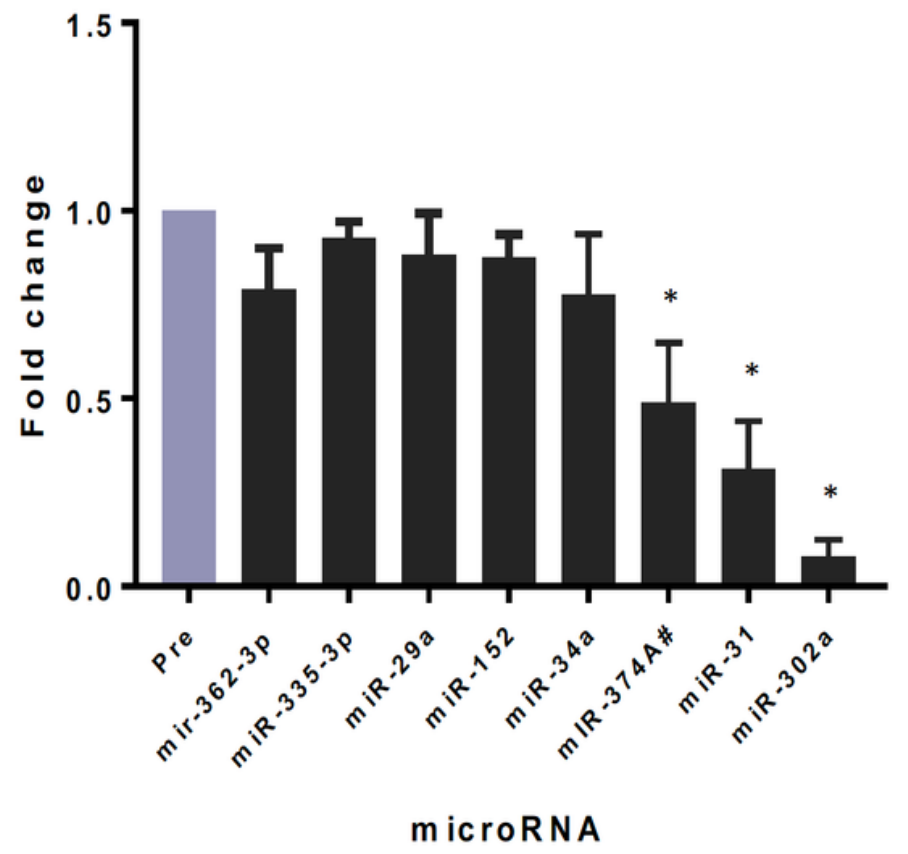

B. Down regulated targets of Axin 1

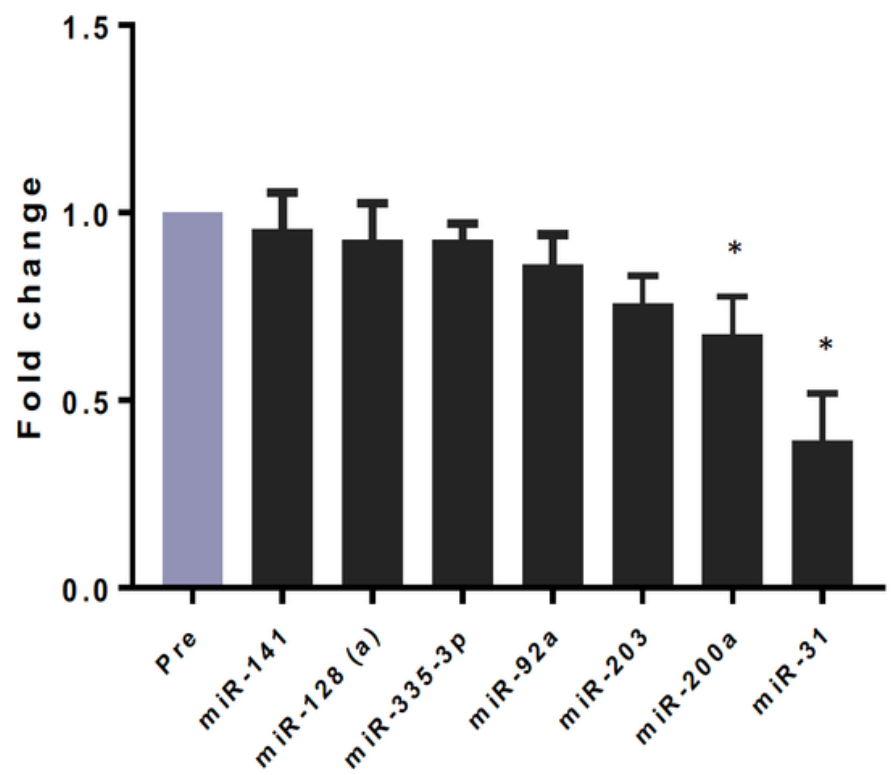

m icroRNA

Figure 7

Potential miRNA targeting genes involved in the Wnt signalling pathway. All data are expressed mean $\pm S E M, n=8$ biological replicates. A Downregulated miRNA which potentially target DKK1; $B$ Downregulated miRNA which potentially target Axin1. The differentially expressed miRNA are shown with an asterisk above them, while other potential targets do not have an asterisk.

\section{Supplementary Files}

This is a list of supplementary files associated with this preprint. Click to download.

- SupplementaryMaterial050721.pdf 